\title{
CO isotopolog line fluxes of viscously evolving disks:
} \section{Cold CO conversion insufficient to explain observed low fluxes}

\author{
L. Trapman ${ }^{1,2}$, A.D. Bosman ${ }^{1,3}$, G. Rosotti ${ }^{1,4}$, M.R. Hogerheijde ${ }^{1,5}$, and E.F. van Dishoeck ${ }^{1,6}$ \\ ${ }^{1}$ Leiden Observatory, Leiden University, Niels Bohrweg 2, NL-2333 CA Leiden, The Netherlands \\ e-mail: ltrapman@wisc.edu \\ 2 Department of Astronomy, University of Wisconsin-Madison, 475 N Charter St, Madison, WI, 53706, USA \\ 3 Department of Astronomy, University of Michigan, 1085 S. University Ave, Ann Arbor, MI 48109, USA \\ 4 School of Physics and Astronomy, University of Leicester, Leicester LE1 7RH, UK \\ 5 Anton Pannekoek Institute for Astronomy, University of Amsterdam, Science Park 904, 1090 GE Amsterdam, The Netherlands \\ ${ }^{6}$ Max-Planck-institute für Extraterrestrische Physik, Giessenbachstraße, D-85748 Garching, Germany
}

Received xx; accepted yy

\begin{abstract}
Context. Protoplanetary disks are thought to evolve viscously, where the disk mass - the reservoir available for planet formation decreases over time as material is accreted onto the central star over a viscous timescale. Observations have shown a correlation between disk mass and the stellar mass accretion rate, as expected from viscous theory. However, this happens only when using the dust mass as a proxy of the disk mass; the gas mass inferred from $\mathrm{CO}$ isotopolog line fluxes, which should be a more direct measurement, shows no correlation with the stellar mass accretion rate.

Aims. We investigate how ${ }^{13} \mathrm{CO}$ and $\mathrm{C}^{18} \mathrm{O} J=3-2$ line fluxes, commonly used as gas mass tracers, change over time in a viscously evolving disk and use them together with gas disk sizes to provide diagnostics of viscous evolution. In addition, we aim to determine if the chemical conversion of $\mathrm{CO}$ through grain-surface chemistry combined with viscous evolution can explain the $\mathrm{CO}$ isotopolog observations of disks in Lupus.

Methods. We ran a series of thermochemical DALI models of viscously evolving disks, where the initial disk mass is derived from observed stellar mass accretion rates.

Results. While the disk mass, $M_{\text {disk }}$, decreases over time, the ${ }^{13} \mathrm{CO}$ and $\mathrm{C}^{18} \mathrm{O} J=3-2$ line fluxes instead increase over time due to their optically thick emitting regions growing in size as the disk expands viscously. The $\mathrm{C}^{18} \mathrm{O} 3-2$ emission is optically thin throughout the disk for only for a subset of our models $\left(M_{*} \leq 0.2 \mathrm{M}_{\odot}\right.$ and $\alpha_{\text {visc }} \geq 10^{-3}$, corresponding to $M_{\text {disk }}\left(t=1 \mathrm{Myr} \leq 10^{-3} \mathrm{M}_{\odot}\right)$. For these disks the integrated $\mathrm{C}^{18} \mathrm{O}$ flux decreases with time, similar to the disk mass. Observed ${ }^{13} \mathrm{CO}$ and $\mathrm{C}^{18} \mathrm{O} 3-2$ fluxes of the most massive disks $\left(M_{\text {disk }} \gtrsim 5 \times 10^{-3} \mathrm{M}_{\odot}\right)$ in Lupus can be reproduced to within a factor of $\sim 2$ with viscously evolving disks in which $\mathrm{CO}$ is converted into other species through grain-surface chemistry with a moderate cosmic-ray ionization rate of $\zeta_{\mathrm{cr}} \sim 10^{-17} \mathrm{~s}^{-1}$. The $\mathrm{C}^{18} \mathrm{O}$ 3-2 fluxes for the bulk of the disks in Lupus (with $M_{\text {disk }} \lesssim 5 \times 10^{-3} \mathrm{M}_{\odot}$ ) can be reproduced to within a factor of $\sim 2$ by increasing $\zeta_{\text {cr }}$ to $\sim 5 \times 10^{-17}-10^{-16} \mathrm{~s}^{-1}$, although explaining the stacked upper limits requires a lower average abundance than our models can produce. In addition, increasing $\zeta_{\text {cr }}$ cannot explain the observed ${ }^{13} \mathrm{CO}$ fluxes for lower mass disks, which are more than an order of magnitude fainter than what is predicted. In our models the optically thick ${ }^{13} \mathrm{CO}$ emission originates from a layer higher up in the disk $(z / r \sim 0.25-0.4)$ where photodissociation stops the conversion of $\mathrm{CO}$ into other species. Reconciling the ${ }^{13} \mathrm{CO}$ fluxes of viscously evolving disks with the observations requires either efficient vertical mixing or low mass disks $\left(M_{\text {dust }} \lesssim 3 \times 10^{-5} \mathrm{M}_{\odot}\right)$ being much thinner and/or smaller than their more massive counterparts.

Conclusions. The ${ }^{13} \mathrm{CO}$ model flux predominantly traces the disk size, but the $\mathrm{C}^{18} \mathrm{O}$ model flux traces the disk mass of our viscously evolving disk models if chemical conversion of $\mathrm{CO}$ is included. The discrepancy between the $\mathrm{CO}$ isotopolog line fluxes of viscously evolving disk models and the observations suggests that $\mathrm{CO}$ is efficiently vertically mixed or that low mass disks are smaller and/or colder than previously assumed.
\end{abstract}

Key words. Protoplanetary disks - Astrochemistry - radiative transfer - line: formation

\section{Introduction}

With over 4200 exoplanets detected ${ }^{1}$ in a multitude of planetary systems, it has become clear that the formation of planets around young stars is a common occurrence (see, e.g., Borucki et al. 2011; Winn \& Fabrycky 2015; Morton et al. 2016). Understanding the processes behind the formation of planets has proven challenging. A key ingredient is the total amount of material available in the protoplanetary disks in which these planets form and grow (see, e.g., Benz et al. 2014, Armitage 2015; Mor-

http: //www . exoplanet . eu dasini 2018). The disk mass determines how much raw material can be accreted onto the forming planets. The disk mass is also not static as accretion onto the central star slowly decreases its mass over time. Combined, the disk mass and the stellar mass accretion rate determine the lifetime of the disk and therefore set an upper limit to the duration of planet formation. Determining disk masses and how they evolve over time is therefore crucial for our understanding of planet formation.

It is commonly assumed that protoplanetary disks evolve viscously (see, e.g., Lynden-Bell \& Pringle 1974; Shakura \& Sunyaev 1973). Viscous stresses in the disk transport angular mo- 
mentum outward, causing the outer parts of the disk to spread out. To conserve angular momentum, this causes material to be transported inward, where it is accreted onto the central star. While still debated, the physical process behind this effective viscosity is commonly assumed to be the magnetorotational instability (see, e.g. Balbus \& Hawley 1991, 1998). In the framework of viscous evolution, the disk mass, $M_{\text {disk }}$, and the stellar mass accretion rate, $\dot{M}_{\text {acc }}$, follow a linear relation, with the disk mass being accreted onto the star on a viscous timescale $\left(M_{\text {disk }} \sim \dot{M}_{\text {acc }} t_{\text {visc }}\right)$.

Alternatively, it has been suggested that the angular momentum in disks can be removed by a magnetic disk wind rather than transported outward by viscous stresses (see, e.g Turner et al. 2014). This magnetic disk wind forms in the presence of a vertical magnetic field in the disk, and it is able to remove material from the disk surface, thus reducing the total angular momentum in the disk. What fraction of the angular momentum can be carried away by the disk wind is still a matter of debate (see, e.g., Ferreira et al. 2006; Béthune et al. 2017; Zhu \& Stone 2018). Magnetic disk winds have been detected in observations but predominantly in the inner part of the disk, and it remains unclear how much disk winds affect disk evolution (see, e.g., Pontoppidan et al.|2011, Bjerkeli et al.|2016; Tabone et al.|2017; de Valon et al. 2020).

Recently, several combined observing campaigns have performed large surveys of the full disk population, allowing the simultaneous study of properties of protoplanetary disks and the young stellar objects that host them. Several star-forming regions have been covered by Atacama Large Millimeter/submillimeter Array (ALMA) disk surveys, providing high angular resolution observations of disk continuum emission and carbon monoxide (CO) rotational line emission (e.g., Ansdell et al. 2016; Ansdell et al. 2017, 2018, Barenfeld et al.|2016, 2017; Pascucci et al. 2016; Long et al. 2017, Cazzoletti et al. 2019, Cox et al. 2017; Cieza et al. 2019, Williams et al. 2019). Using spectra from the X-shooter spectrograph (Vernet et al. 2011) at the ESO Very Large Telescope, stellar properties such as the stellar mass accretion rate have also been measured for a large fraction of the diskhosting stars in star-forming regions such as Lupus, Chamaeleon I, and Upper Sco (Alcalá et al.2014, 2017, Manara et al. 2017, 2020).

Combining observations for the disk population in Lupus, Manara et al. (2016) found a correlation between the observed stellar mass accretion rate, $\dot{M}_{\text {acc }}$, and the disk dust mass, $M_{\text {dust }}$, derived from millimeter continuum emission. If an interstellar medium (ISM) gas-to-dust mass ratio $\Delta_{\mathrm{gd}}=100$ is assumed, the observations $\left(\dot{M}_{\text {acc }}\right.$ and $\left.M_{\text {disk }}=100 \times M_{\text {dust }}\right)$ are consistent with viscous disks having evolved for 1-3 Myr, which matches the approximate age of the sources (see also Rosotti et al. 2017). Interestingly, they find no correlation between $M_{\text {acc }}$ and the disk gas mass, $M_{\text {gas }}$, derived from ${ }^{13} \mathrm{CO} J=3-2$ and $\mathrm{C}^{18} \mathrm{O} J=3-2$ line fluxes. This seems to contradict their first finding, suggesting that the disk gas mass, which is expected to make up most of the total disk mass, is not related to the stellar mass accretion rate.

The cause for this discrepancy might lie with the tracer used to measure the disk gas mass. For most disks the gas masses derived from optically thin $\mathrm{CO}$ isotopologs such as ${ }^{13} \mathrm{CO}$ and $\mathrm{C}^{18} \mathrm{O}$ are found to be low compared to their dust mass, with $\Delta_{\mathrm{gd}}=M_{\mathrm{gas}} / M_{\text {dust }} \approx 1-10$ for most disks (see, e.g., Ansdell et al. 2016, Miotello et al. 2017, Long et al. 2017). Herschel Space observatory observations of the hydrogen deuteride (HD) $J=1-0$ rotational line toward a handful of disks have provided an independent measurement of the disk gas mass (Bergin et al. 2013; McClure et al. 2016, Trapman et al. 2017, Kama et al. 2020). These observations find a gas-to-dust mass ratio of $\sim 100$, suggesting that the low CO-based gas masses are a sign that disks are underabundant in $\mathrm{CO}$. This underabundance is in addition to well-understood processes such as $\mathrm{CO}$ freeze-out and photodissociation. Several processes have been suggested to explain the extra underabundance of $\mathrm{CO}$, such as chemical conversion of $\mathrm{CO}$ in the gas or on the grains into more complex species (e.g., Aikawa et al. 1997; Bergin et al. 2014; Furuya \& Aikawa 2014; Yu et al. 2016, 2017; Dodson-Robinson et al. 2018, Bosman et al. 2018; Schwarz et al. 2018) or the locking of CO in larger bodies (see, e.g., Bergin et al. 2010; Bergin et al. 2016, Kama et al. 2016; Krijt et al. 2018).

In this work we use an alternative approach to investigate the lack of a correlation between $\dot{M}_{\text {acc }}$ and the CO-based $M_{\text {gas }}$, by taking a step back and examine what ${ }^{13} \mathrm{CO}$ and $\mathrm{C}^{18} \mathrm{O}$ line fluxes are expected for viscously evolving disks. Over time the disk spreads out, increasing the emitting region, and the disk mass decreases, lowering the ${ }^{13} \mathrm{CO}$ - and $\mathrm{C}^{18} \mathrm{O}$-column densities, both of which affect the resulting line fluxes. Furthermore, by using the initial gas masses that can explain the observed stellar mass accretion rates, we can examine if the observed ${ }^{13} \mathrm{CO}$ and $\mathrm{C}^{18} \mathrm{O}$ line fluxes are consistent with viscous evolution.

Recently, Trapman et al. (2020) used the same modeling framework to examine if observed gas outer radii of disks in the Lupus and Upper Sco star-forming regions can be explained with viscous evolution (see also Ansdell et al. 2018; Barenfeld et al. 2017). They showed that gas outer radii of disks in Lupus are consistent with viscously evolving disks that start out small, meaning an initial characteristic radius of $\sim 10 \mathrm{AU}$, and that have a low viscosity $\left(\alpha_{\text {visc }}=10^{-4}-10^{-3}\right)$. Combining their results with our analysis of the $\mathrm{CO}$ isotopolog line fluxes, we can examine if disks are in agreement with viscous theory in terms of both their size and their mass.

The structure of this work is as follows: In Section 2 we discuss the setup and initial conditions of our models. Here we also outline how we implement the chemical conversion of $\mathrm{CO}$ through grain-surface chemistry. In Section 3 we first show how ${ }^{13} \mathrm{CO}$ and $\mathrm{C}^{18} \mathrm{O}$ intensity profiles and integrated line fluxes change over time in a viscously evolving disk and how they shift if grain-surface chemistry converts $\mathrm{CO}$ into other species. Next we compare our models to observations in Lupus and discuss the cosmic-ray ionization rates that are required to match the observed fluxes. In Section 4 we look in more detail at the ${ }^{13} \mathrm{CO}$ and $\mathrm{C}^{18} \mathrm{O}$ fluxes that are overproduced by our models and we discuss the impact of vertical mixing and alternative explanations such as small disks. We conclude in Section 5 that reconciling the ${ }^{13} \mathrm{CO}$ and $\mathrm{C}^{18} \mathrm{O}$ fluxes of viscously evolving disks models with the observations requires either that $\mathrm{CO}$ is efficiently mixed vertically or that low mass disks are small.

\section{Model setup}

For setting up the initial conditions and time evolution of our models, we follow the same approach as presented Trapman et al. (2020). For completeness we reiterate the steps in this approach here.

Based on observed stellar accretion rates we calculate what the initial disk mass must have been, assuming that the disk has evolved viscously. From the initial disk masses we compute the surface density profile analytically at 10 consecutive disk ages. At each time step the thermochemical code Dust and Lines (DALI; Bruderer et al. 2012, Bruderer 2013) is used to calculate the abundances, excitation and temperature of the disk and the model is ray-traced to obtain ${ }^{13} \mathrm{CO} J=3-2$ and $\mathrm{C}^{18} \mathrm{O}$ 
$J=3-2$ line fluxes. This modeling approach was previously used by Trapman et al. (2020) to study the evolution of measured gas disk sizes.

\subsection{Viscous evolution of the surface density}

While the physical processes underlying the viscosity in disks are still an open question, it is common to describe the kinetic viscosity $v$ using the dimensionless parameter $\alpha_{\text {visc }}$, defined as $v=\alpha c_{s} H$, where $c_{s}$ is the sound speed and $H$ is the height above the midplane (the $\alpha$-disk formalism, see Shakura \& Sunyaev 1973; Pringle 1981). In this formalism a self-similar solution for the surface density $\Sigma$ can be calculated (Lynden-Bell \& Pringle 1974; Hartmann et al. 1998)

$$
\Sigma_{\text {gas }}(R)=\frac{(2-\gamma) M_{\mathrm{disk}}(t)}{2 \pi R_{\mathrm{c}}(t)^{2}}\left(\frac{R}{R_{\mathrm{c}}(t)}\right)^{-\gamma} \exp \left[-\left(\frac{R}{R_{\mathrm{c}}(t)}\right)^{2-\gamma}\right],
$$

where $\gamma$ enters by assuming that the viscosity varies radially as $v \propto R^{\gamma}$ and $M_{\text {disk }}$ and $R_{\mathrm{c}}$ are the disk mass and the characteristic radius, respectively.

The evolution of the surface density depends on how $M_{\text {disk }}$ and $R_{\mathrm{c}}$ change over time (see, e.g., Hartmann et al. 1998):

$$
\begin{aligned}
M_{\text {disk }}(t) & =M_{\text {disk }}(t=0)\left(1+\frac{t}{t_{\text {visc }}}\right)^{-\frac{1}{[2(2-\gamma)]}} \\
& =M_{\text {disk }}(t=0)\left(1+\frac{t}{t_{\text {visc }}}\right)^{-\frac{1}{2}} \\
R_{\mathrm{c}}(t) & =R_{\mathrm{c}}(t=0)\left(1+\frac{t}{t_{\mathrm{visc}}}\right)^{\frac{1}{(2-\gamma)}} \\
& =R_{\mathrm{c}}(t=0)\left(1+\frac{t}{t_{\mathrm{visc}}}\right) .
\end{aligned}
$$

Here $t_{\text {visc }}$ is the viscous timescale. For the second step and in the rest of this work we have assumed $\gamma=1$. For a typical temperature profile this corresponds to the case where $\alpha_{\text {visc }}$ stays constant with radius. The time evolution of $M_{\text {disk }}$ for our models can be seen in Figure 1 .

\subsection{Initial disk mass and disk size}

Over time the mass of a disk decreases as material is accreted onto the central star. For a disk that is viscously evolving with a constant $\alpha_{\text {visc }}$ the relation between the initial disk mass $M_{\text {disk }}(t=$ 0 ) and the stellar mass accretion rate, $\dot{M}_{\text {acc }}$, at a given time $t$ can be written such that the initial disk mass is a function of $\dot{M}_{\text {acc }}(\mathrm{t})$ (see, e.g., Hartmann et al. 1998)

$M_{\text {disk }}(t=0)=2 t_{\text {visc }} \dot{M}_{\text {acc }}(t)\left(\frac{t}{t_{\text {visc }}}+1\right)^{3 / 2}$.

Stellar mass accretion rates have now been measured for multiple star-forming regions such as Lupus, Chamaeleon I, and Upper Sco (Alcalá et al. 2014, 2017, Manara et al. 2017, 2020). Based on these accretion rates we can calculate what the initial disk masses would have been, given the viscous timescale and the average age of the star-forming region.

To determine the initial disk masses of the models, our approach is as follows. We take four stellar mass accretion rates $\left(4 \times 10^{-12}, 4 \times 10^{-11}, 2 \times 10^{-9}\right.$ and $\left.10^{-8} \mathrm{M}_{\odot} \mathrm{yr}^{-1}\right)$ that span

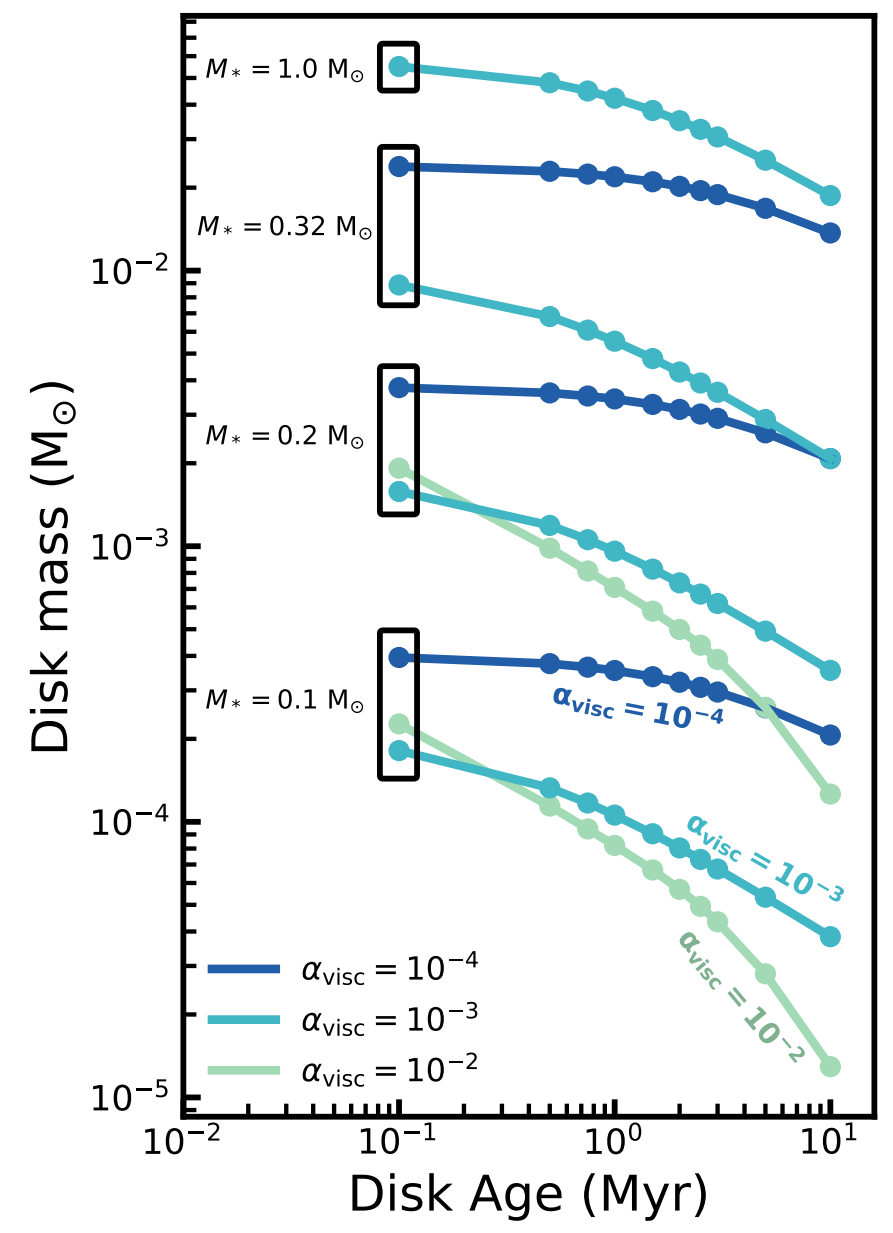

Fig. 1: Time evolution of the disk mass, $M_{\text {disk }}$, of our models. Colors indicate the viscous alpha, $\alpha_{\text {visc }}$, of the models. The black boxes show which stellar mass, and therefore accretion rate, was used to calculate the initial disk mass of the models (see Table 1). We note that we exclude models with $\alpha_{\text {visc }}=10^{-2}$ for $M_{*}=$ $[0.32,1.0] \mathrm{M}_{\odot}$ as the gas disk sizes of these models can be ruled out by observations of disks in Lupus (Trapman et al. 2020). We also note that models with $M_{*}=1.0 \mathrm{M}_{\odot}$ and $\alpha_{\text {visc }}=10^{-4}$ are much more massive $\left(M_{\text {gas }} \gtrsim 2 \times 10^{-1} \mathrm{M}_{\odot}\right)$ than the disks observed in Lupus (cf. Ansdell et al. 2016; see Figure A.1 in Trapman et al. 2020).

the range of the observations in Lupus (see, e.g., Alcalá et al. 2014, 2017). As observations have shown that $\overline{M_{\text {acc }}}$ is correlated to $M_{*}$, our selected $\dot{M}_{\text {acc }}$ represent the average accretion rates for stars with $M_{*}=0.1,0.2,0.32$, and $1.0 \mathrm{M}_{\odot}$. Compared to Trapman et al. (2020) this includes one more set of models $\left(M_{*}=0.2 \mathrm{M}_{\odot}\right)$. For these stellar mass accretion rates we calculate what the initial disk mass must have been for three different viscous timescales, assuming an average age of $1 \mathrm{Myr}$ for Lupus. The viscous timescales are calculated for three values of the dimensionless viscosity $\alpha_{\text {visc }}=10^{-2}, 10^{-3}, 10^{-4}$, assuming a characteristic radius of $10 \mathrm{AU}$ (a choice that is discussed below) and a disk temperature $T_{\text {disk }}$ of $20 \mathrm{~K}$ (see, e.g., equation 37 in Hartmann et al. 1998)

$$
\frac{t_{\mathrm{visc}}}{\mathrm{yr}}=\frac{R_{\mathrm{c}}^{2}}{v} \simeq 8 \times 10^{4}\left(\frac{10^{-2}}{\alpha_{\mathrm{visc}}}\right)\left(\frac{R_{\mathrm{c}}}{10 \mathrm{AU}}\right)\left(\frac{M_{*}}{0.5 \mathrm{M}_{\odot}}\right)^{1 / 2}\left(\frac{10 \mathrm{~K}}{T_{\text {disk }}}\right) .
$$


(7)

The resulting initial disk masses, 12 in total, are summarized in Table 1. Figure 1 shows how these disk masses decrease over time. In our analysis we exclude three models. Trapman et al. (2020) showed that the gas disk sizes measured from models with $\alpha=10^{-2}$ and $M_{*}=[0.32,1.0] \mathrm{M}_{\odot}$ can be ruled out based on observed gas disk sizes in Lupus. We also exclude the model with $M_{*}=1.0 \mathrm{M}_{\odot}$ and $\alpha=10^{-4}$, which has an initial disk mass of $M_{\text {disk }}=2.6 \times 10^{-1} \mathrm{M}_{\odot}$. At all points during its evolution, this model is factor of five more massive than the most massive disk observed in Lupus, but consistent with Class 0/1 disk masses (cf. Figure A.1 in Trapman et al. 2020, see also Tychoniec et al. 2018, 2020; Tobin et al. 2020).

For the initial disk size we assume that disks start out small, with an initial characteristic disk size $R_{\mathrm{c}}(t=0)=10 \mathrm{AU}$. Recently, Trapman et al. (2020) showed that the observed gas outer radii of Class II disks in Lupus can be explained by viscously evolving disks that start out small $\left(R_{\mathrm{c}}(t=0)=10 \mathrm{AU}\right)$ and that have a low viscosity $\left(\alpha_{\text {visc }}=10^{-4}-10^{-3}\right)$. More importantly, they show that the bulk of the observed gas outer radii cannot be explained by disks that start out large $\left(R_{\mathrm{c}}(t=0) \geq 30 \mathrm{AU}\right)$.

Observational constraints on the disk size of young Class 1 and 0 objects have only recently become available. In their VANDAM II survey, Tobin et al. 2020 presented ALMA 0.87 millimeter observations of 330 protostars in Orion at a resolution of $\sim 0$ '”1 ( $\sim 40 \mathrm{AU}$ in diameter). Their observations suggest that the majority of disks are initially small $(\sim 37-45 \mathrm{AU}$ in radius), at least as seen in the emission of the millimeter dust. It is worth mentioning that their radii are defined as half of the full with at half maximum (FWHM) of a 2D Gaussian fit to the observations, which is not the same as the characteristic radius of the disk. For the gas there is similar evidence that disks start out small, albeit from a smaller sample. Maret et al. (2020) presented NOEMA observations of 16 Class 0 protostars as part of the CALYPSO large program. They found only two sources that show a Keplerian disk larger than $\sim 50$ AU. This suggests that stars with a large Keplerian disk at a young age, such as those found for VLA 1623 (Murillo et al. 2013), are uncommon. We therefore adopted an initial disk size of $R_{\text {init }}=10 \mathrm{AU}$ for our models.

Table 1: Initial conditions of our DALI models

\begin{tabular}{lc|ccc}
\hline \hline $\begin{array}{l}M_{*} \\
\left(\mathrm{M}_{\odot}\right)\end{array}$ & $\begin{array}{c}\dot{M}_{\mathrm{acc}} \\
\left(\mathrm{M}_{\odot} \mathrm{yr}^{-1}\right)\end{array}$ & $10^{-2}$ & $\begin{array}{c}\alpha_{\text {visc }} \\
10^{-3}\end{array}$ & $10^{-4}$ \\
\hline & & \multicolumn{3}{|c}{$M_{\text {disk }}\left(\mathrm{M}_{\odot}\right)$ at $t=0$} \\
\hline 0.1 & $4 \times 10^{-11}$ & $4.5 \times 10^{-4}$ & $2.1 \times 10^{-4}$ & $4.1 \times 10^{-4}$ \\
0.2 & $4 \times 10^{-10}$ & $3.6 \times 10^{-3}$ & $1.8 \times 10^{-3}$ & $3.9 \times 10^{-3}$ \\
0.32 & $2 \times 10^{-9}$ & $2.0 \times 10^{-2}$ & $1.0 \times 10^{-2}$ & $2.5 \times 10^{-2}$ \\
1.0 & $1 \times 10^{-8}$ & $6.9 \times 10^{-2}$ & $5.9 \times 10^{-2}$ & $2.6 \times 10^{-1}$ \\
\hline
\end{tabular}

Notes. Disk masses in italic are not included in our analysis (see Section 2.2. The viscous timescale in the models varies approximately as $t_{\text {visc }} \simeq 0.5 \times\left(10^{-3} / \alpha_{\text {visc }}\right) \times 10^{6} \mathrm{yr}$.

\subsection{The DALI models}

Using the initial conditions discussed previously we computed how $M_{\text {disk }}$ decreases and $R_{\mathrm{c}}$ increases over time. For ten points in the lifetime of the disk, starting at $0.1 \mathrm{Myr}$ and ending at 10 Myr, we computed the current surface density $\Sigma_{\text {gas }}(t)$ of the disk model.

We used the thermochemical code DALI to compute CO isotopolog abundances and line fluxes of our disk models. DALI is a 2D physical-chemical code that computes the thermal and chemical structure for a given physical disk structure. For each $\Sigma_{\text {gas }}(t)$ a Monte Carlo radiative transfer calculation is used determine the dust temperature and the internal radiation field from UV- to mm-wavelengths. Next, the code computes atomic and molecular abundances by solving the time-dependent chemistry. The atomic and molecular excitation is then obtained using a nonLTE calculation. The heating and cooling balance is solved to calculate the gas temperature. These three steps were performed iteratively until a self-consistent solution is obtained. Raytracing of the model then yields line fluxes. For a more detailed description of the code we refer the reader to Appendix A of Bruderer et al. (2012).

To obtain a 2D (i.e., $R, z$ ) density structure for our models, we assumed that in the vertical direction the disk follows a Gaussian density distribution, as motivated by hydrostatic equilibrium (see, e.g., Chiang \& Goldreich 1997). The height of the disk was assumed to follow a powerlaw of the form $H=R h=$ $R h_{c}\left(R / R_{\mathrm{c}}\right)^{\psi}$, where $h_{c}$ is the opening angle at $R_{\mathrm{c}}$ and $\psi$ is the flaring angle.

As grains grow larger they decouple from the gas and settle toward the midplane of the disk (see, e.g., Dubrulle et al. 1995. Dullemond \& Dominik 2004, 2005, Mulders \& Dominik 2012, Riols \& Lesur 2018). The settling of large grains is included in our models in a simplified way by splitting the dust grains into two populations, following the approach in Andrews et al. (2011). Most of the dust mass (90\%) is in large grains with sizes of $1-10^{3} \mu \mathrm{m}$. These large grains follow the gas radially, but vertically they are confined to the midplane as their scale height is reduced by a factor $\chi=0.2$ with respect to the gas. A population of small grains $(0.005-1 \mu \mathrm{m})$ make up the remaining $10 \%$ of the dust mass in the disk. These small grains follow the distribution of the gas both radially and vertically.

We assume that our disks encircle a typical T-Tauri star, which we represent with a blackbody with effective temperature for $T_{\text {eff }}=4000 \mathrm{~K}$. A second blackbody with $T_{\text {eff }}=10000 \mathrm{~K}$ is added to simulate the excess UV radiation released by material accreted onto the star. To compute the luminosity of this second component we follow Kama et al. (2015) and assume that the gravitational potential energy of the accreted material is converted into radiation with a $100 \%$ efficiency. These parameters are summarized in Table 2. We note that stellar evolution is not included in our models. Including stellar evolution can affect CO chemistry and its chemical conversion. Especially the higher stellar luminosity early on will increase the disk temperature and reduce the amount of $\mathrm{CO}$ freeze out (see e.g., the observations presented in van 't Hoff et al. 2020; van't Hoff et al. 2020). A detailed study of the effect of stellar evolution on CO chemistry is available in Yu et al. (2016).

\subsubsection{Isotopolog chemical network}

For less abundant isotopologs of $\mathrm{CO}$ such as ${ }^{13} \mathrm{CO}, \mathrm{C}^{18} \mathrm{O}$, and $\mathrm{C}^{17} \mathrm{O}$, isotope-selective photodissociation can have significant effect on their abundances (see, e.g., Visser et al. 2009, Miotello et al. 2014). Using the results from Visser et al. 2009, Miotello et al. (2014) expanded the standard chemical network of DALI, based on the UMIST 06 network (Woodall et al. 2007; Bruderer et al. 2012, Bruderer 2013), to include the isotope-selective 
Table 2: DALI parameters of the physical model.

\begin{tabular}{|c|c|}
\hline Parameter & Range \\
\hline \multicolumn{2}{|l|}{ Chemistry } \\
\hline Chemical age & $0.1-10 \mathrm{Myr}^{*, \dagger}$ \\
\hline Volatile $[\mathrm{C}] /[\mathrm{H}]$ & $1.35 \times 10^{-4}$ \\
\hline Volatile $[\mathrm{O}] /[\mathrm{H}]$ & $2.88 \times 10^{-4}$ \\
\hline \multicolumn{2}{|l|}{ Physical structure } \\
\hline$\gamma$ & 1.0 \\
\hline$\psi$ & 0.15 \\
\hline$h_{c}$ & 0.1 \\
\hline$R_{\mathrm{c}}$ & $10-3 \times 10^{3} \mathrm{AU}^{\dagger}$ \\
\hline$M_{\mathrm{gas}}$ & $10^{-5}-10^{-1} \mathrm{M}_{\odot}^{\dagger}$ \\
\hline Gas-to-dust ratio & 100 \\
\hline \multicolumn{2}{|l|}{ Dust properties } \\
\hline$f_{\text {large }}$ & 0.9 \\
\hline$\chi$ & 0.2 \\
\hline composition & standard ISM $^{1}$ \\
\hline$f_{\mathrm{PAH}}$ & 0.001 \\
\hline \multicolumn{2}{|l|}{ Stellar spectrum } \\
\hline$T_{\text {eff }}$ & $4000 \mathrm{~K}+$ Accretion U \\
\hline$L_{*}$ & $0.28 \mathrm{~L}_{\odot}$ \\
\hline$L_{\mathrm{X}}$ & $10^{30} \mathrm{erg} \mathrm{s}^{-1}$ \\
\hline$T_{X}$ & $3.2 \times 10^{6} \mathrm{~K}$ \\
\hline$\zeta_{\mathrm{cr}}$ & $10^{-17} \mathrm{~s}^{-1}$ \\
\hline \multicolumn{2}{|l|}{ Observational geometry } \\
\hline$i$ & $0^{\circ}$ \\
\hline PA & $0^{\circ}$ \\
\hline$d$ & $150 \mathrm{pc}$ \\
\hline
\end{tabular}

Notes. * The age of the disk is taken into account when running the time-dependent chemistry. ${ }^{\dagger}$ These parameters evolve with time. " Weingartner \& Draine 2001. see also Section 2.5 in Facchini et al. 2017 .

photodissociation and chemistry of ${ }^{13} \mathrm{CO}, \mathrm{C}^{18} \mathrm{O}$, and $\mathrm{C}^{17} \mathrm{O}$. The chemical network includes the formation of $\mathrm{H}_{2}$ on grains, freezeout, thermal and nonthermal desorption of species, hydrogenation of simple species on ices, gas-phase reactions, photodissociation, X-ray- and cosmic-ray-induced reactions, polycyclic aromatic hydrocarbon (PAH) grain charge exchange and/or hydrogenation, and reactions with vibrationally excited $\mathrm{H}_{2}^{*}$. A detailed description of the implementation of these reactions can be found in Appendix A.3.1 of Bruderer et al. (2012). A full description of the isotopolog chemical network can be found in Miotello et al. (2014). For the initial volatile carbon and oxygen abundances, typical ISM values of $[\mathrm{C}] /[\mathrm{H}]_{\text {ISM }}=1.35 \cdot 10^{-4}$, $[\mathrm{O}] /[\mathrm{H}]_{\mathrm{ISM}}=2.88 \cdot 10^{-4}$ were used (Cardelli et al. 1996, Jonkheid et al. 2007; Woitke et al. 2009; Bruderer et al. 2012). We take the isotope ratios to be $\left[{ }^{12} \mathrm{C}\right] /\left[{ }^{13} \mathrm{C}\right]=77$ and $\left[{ }^{16} \mathrm{O}\right] /\left[{ }^{18} \mathrm{O}\right]$ $=560$ (Wilson \& Rood 1994).

Relevant for this work is that in the DALI chemical network $\mathrm{CO}$ is not further processed once it is frozen out on the grains. The only reaction included in the network that affects $\mathrm{CO}$ ice is desorption back into the gas phase.

\subsubsection{CO conversion through grain-surface chemistry}

Recent observations have shown that a large fraction of protoplanetary disks have unexpectedly low ${ }^{13} \mathrm{CO}$ and $\mathrm{C}^{18} \mathrm{O}$ line fluxes (see, e.g., Ansdell et al. 2016, Miotello et al. 2017, Long et al. 2017). Gas masses derived from these line fluxes using

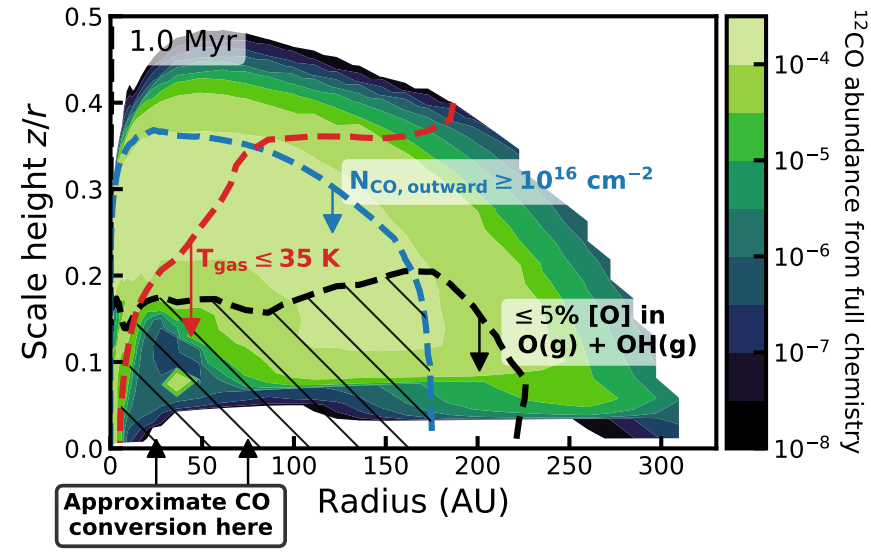

Fig. 2: CO abundance computed using the full chemical network in Bosman et al. (2018, see also Cridland et al. 2020) for the model with $M_{*}=0.32 \mathrm{M}_{\odot}$ and $\alpha_{\text {visc }}=10^{-3}$ at $1 \mathrm{Myr}$. The chemistry was calculated using a $\zeta_{\mathrm{cr}}=10^{-16} \mathrm{~s}^{-1}$ to show where $\mathrm{CO}$ can be transformed. Taken from the same model, but with the chemistry calculated within DALI, the dashed blue line shows where the CO outward column is $10^{16} \mathrm{~cm}^{-2}$. The black contour shows where $5 \%$ of the total oxygen abundance is in $\mathrm{OH}(\mathrm{g})+$ $\mathrm{O}(\mathrm{g})$. The red contour shows where $T_{\mathrm{gas}}=35 \mathrm{~K}$. The hatched region below the three dashed lines shows where the $\mathrm{CO}$ abundance is recalculated using the approximate grain-surface chemistry.

models that include freeze-out and photodissociation suggest that the bulk of the protoplanetary disks are gas poor, with gasto-dust mass ratios $\left(\Delta_{\mathrm{gd}}\right)$ on the order of $\Delta_{\mathrm{gd}} \approx 1-10$. For a few disks the gas mass has been determined independently using HD (see, e.g., Bergin et al. 2013, McClure et al.2016; Trapman et al. 2017, Kama et al. 2020). These observations suggest instead that $\mathrm{CO}$ is underabundant in disks (see, e.g., Favre et al. 2013; $\mathrm{Du}$ et al. 2015; Bergin et al. 2016, Kama et al. 2016, Zhang et al. 2019). This requires some process currently not accounted for that removes $\mathrm{CO}$ from the gas phase. Two such processes have been suggested: The first proposes that when $\mathrm{CO}$ freezes out on grain it can become locked up in larger bodies, preventing it from reentering the gas phase (see, e.g., Bergin et al. 2010, Bergin et al. 2016, Du et al. 2015, Kama et al. 2016, Krijt et al. 2018, 2020). Radial drift of these larger bodies can move the frozen out CO to smaller radii (see, e.g Booth et al.2017). Several authors have also shown that grain-surface chemistry is capable of lowering the $\mathrm{CO}$ abundance in disks. In the gas collisions with $\mathrm{He}^{+}$ can break apart $\mathrm{CO}$ molecules, allowing the available carbon to be locked up in hydrocarbons such as $\mathrm{CH}_{4}$ and $\mathrm{C}_{2} \mathrm{H}_{6}$ (see, e.g., Aikawa et al. 1997, Bergin et al. 2014, Schwarz et al. 2018). In the ice $\mathrm{CO}$ can be converted into other species such as $\mathrm{CO}_{2}$ and $\mathrm{CH}_{3} \mathrm{OH}$ on a timescale of several megayears (see, e.g., Bergin et al. 2014; Yu et al. 2016, 2017, Bosman et al. 2018; Schwarz et al. 2019).

Calculating the "chemical conversion of CO" requires a large chemical network. As expanding the network significantly increases the computation time of a thermochemical model, it is unfeasible to calculate the chemical conversion of $\mathrm{CO}$ for a large set of models. In this work we have instead implemented the chemical conversion of $\mathrm{CO}$ using an approximate description for $\mathrm{CO}$ gas and grain-surface chemistry based on the results of Bosman et al. (2018). The description simplifies the full chemical network in Bosman et al. (2018) by only tracing the main 
without chemical conversion of $\mathrm{CO}$

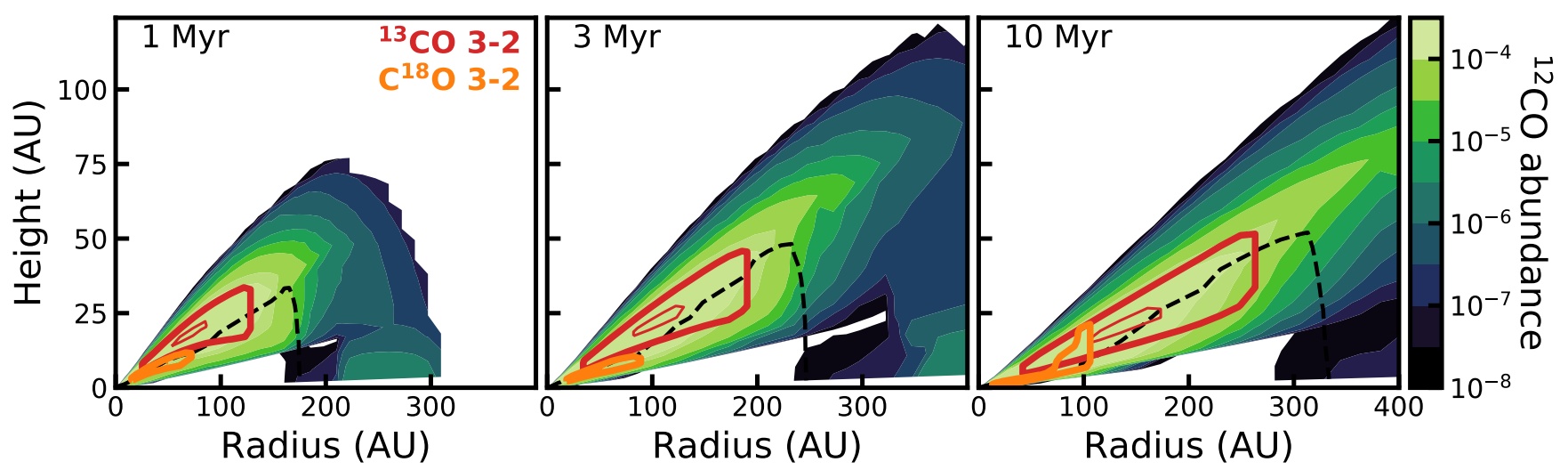

with chemical conversion of $\mathrm{CO}$

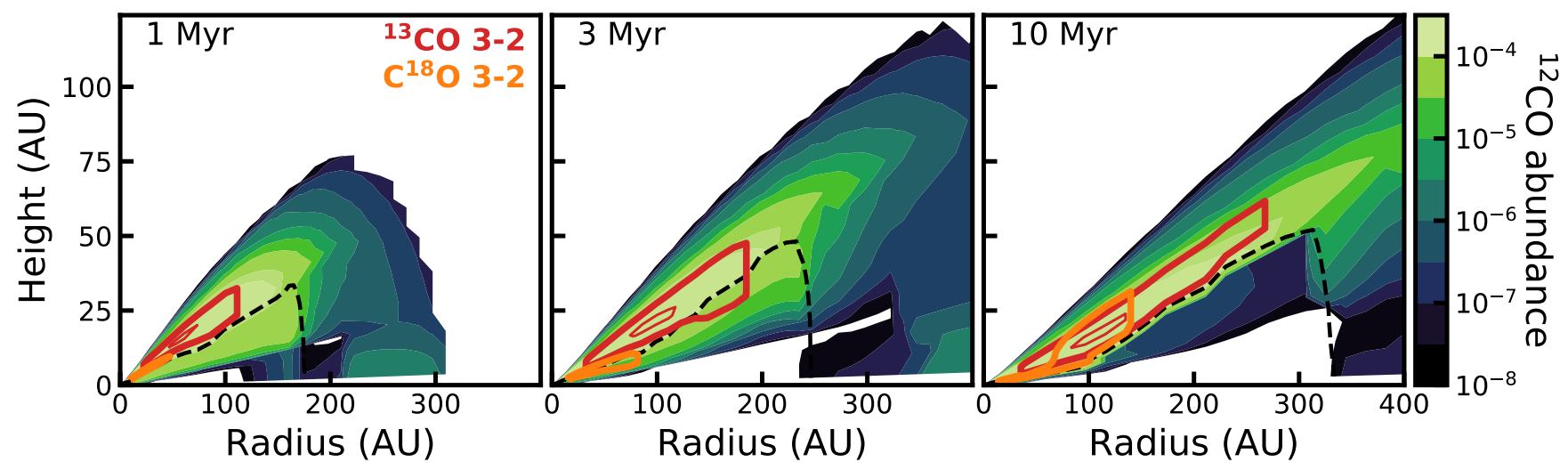

Fig. 3: Effect of the chemical conversion of $\mathrm{CO}$ on the $\mathrm{CO}$ abundance after 1, 3 and 10 Myr. The example model shown here has $M_{*}=0.32 \mathrm{M}_{\odot}$ and $\alpha_{\mathrm{visc}}=10^{-3}$. In the top panels CO abundances have been calculated using the DALI chemical network (see Section 2.3.1). In the bottom panels we have included the chemical conversion of CO into other species, as described in Section 2.3.2. Colors show the $\mathrm{CO}$ abundance with respect to $\mathrm{H}_{2}$, where white indicates $\mathrm{CO} / \mathrm{H}_{2} \leq 10^{-8}$. The dashed black line shows where $\leq 5 \%$ of the total amount of oxygen is in $\mathrm{O}(\mathrm{g})+\mathrm{OH}(\mathrm{g})$, the gas temperature $T_{\text {gas }} \leq 35 \mathrm{~K}$ and outward CO column is $\geq 10^{16}$ $\mathrm{cm}^{-2}$. Grain-surface chemistry is calculated below this black dashed line. Red contours show the ${ }^{13} \mathrm{CO} J=3-2$ emitting region, enclosing $25 \%$ and $75 \%$ of the total ${ }^{13} \mathrm{CO}$ flux. Similarly, the orange contours show the $\mathrm{C}^{18} \mathrm{O} J=3-2$ emitting region.

carbon carriers, meaning $\mathrm{CO}, \mathrm{CH}_{3} \mathrm{OH}, \mathrm{CO}_{2}$ and $\mathrm{CH}_{4}$. The chemistry is split up into the carrier species that have long $\left(>10^{4} \mathrm{yr}\right)$ chemical timescales and intermediates, radicals and ions, which have a short, $<100 \mathrm{yr}$, chemical timescales. The former are explicitly integrated while kinetic equilibrium is assumed for the latter. A more detailed description can be found in Appendix A in Krijt et al. (2020).

Using this approximate grain-surface chemistry, we recalculate the $\mathrm{CO}$ abundances computed by DALI. In doing so some thought has to be put in where in the disk our method gives an accurate approximation of the full chemical network in Bosman et al. (2018). Based on tests where the full chemistry is computed, we identify three boundaries beyond which the shielded midplane approximation is no longer valid: a low outward column of $\mathrm{CO}$, the presence of oxygen in the gas phase, and a high temperature. The first is an outward column of $\mathrm{CO}$ of $N_{\text {CO,outward }}=10^{16} \mathrm{~cm}^{-2}$ (see also van Dishoeck \& Black 1988; Heays et al. 2017). The outward column is defined as the minimum of the vertical and radial external columns. In regions with a lower outward column CO can be photodissociated by UV photons and the $\mathrm{CO}$ abundance is set by the balance between photodissociation and chemistry, which is included in DALI.

The second boundary is related to the fraction of oxygen in the gas phase. The approximation assumes that all gas-phase oxygen directly transforms into $\mathrm{OH}$ ice where it can react with $\mathrm{CO}$ or $\mathrm{H}$ to form $\mathrm{CO}_{2}$ ice or $\mathrm{H}_{2} \mathrm{O}$ ice, respectively. The presence of atomic oxygen in the gas phase in the model indicates that photodissociation is still a significant driver of the chemistry. UV dissociation reactions will dissociate carbon carriers other than $\mathrm{CO}$ and push carbon back into the more photo-stable $\mathrm{CO}$ (see, e.g., van Dishoeck \& Black 1988). Empirically we have found the boundary where $5 \%$ of the available oxygen exists as gasphase $\mathrm{O}$ or $\mathrm{OH}$ encloses the region where in the full chemical network $\mathrm{CO}$ is transformed into other species (see Figure 2).

Finally we also exclude the parts of the disk where $T_{\text {gas }}>$ $35 \mathrm{~K}$. Tests show that above this temperature the conversion of $\mathrm{CO}$ through grain-surface chemistry is negligible. At these temperatures $\mathrm{CO}$ is converted in $\mathrm{CH}_{4}$ in the gas phase (see, e.g., Aikawa et al. 1997; Schwarz et al. 2018. This is the dominant route above $\sim 25 \mathrm{~K}$ and densities below $\lesssim 10^{10} \mathrm{~cm}^{-3}$. It becomes less and less effective with increasing temperature due to the lower sticking of atomic oxygen on grains (see Figure 4 in Bosman et al. 2018). The increased time atomic oxygen spends in the gas phase results in the reformation of $\mathrm{CO}$ through reactions of $\mathrm{O}$ and $\mathrm{OH}$ with hydrocarbon radicals and ions, for example: $\mathrm{CH}_{2}^{+}+\mathrm{O} \rightarrow \mathrm{HCO}^{+} \rightarrow \mathrm{CO}$.

It should be noted that efficiency of gas-phase conversion of $\mathrm{CO}$ depends directly on rate at which $\mathrm{CO}$ can be destroyed 
by $\mathrm{He}^{+}$. Changes to the assumptions made to calculate this rate, for example increasing cosmic-ray ionization rate of $\mathrm{He}$, could increase the efficiency of gas-phase $\mathrm{CO}$ conversion. It should also be noted that our chemical conversion of $\mathrm{CO}$ focuses on the reactions that result in $\mathrm{CO}$ no longer being the main carbon carrier in the disk. Other reactions that include $\mathrm{CO}$ do still occur both inside and outside our set boundaries (e.g., the formation of $\mathrm{H}_{2} \mathrm{CO}$; see, e.g., Loomis et al. 2015, Öberg et al. 2017; Terwisscha van Scheltinga et al. 2020), but these reactions do not significantly affect $\mathrm{CO}$ being the predominant carbon carrier. To summarize, $\mathrm{CO}$ abundances are recalculated using the approximate grain-surface chemistry for regions in the disk where $N_{\mathrm{CO} \text {,outward }}>10^{16}, \leq 5 \%$ of $[\mathrm{O}]$ is in $\mathrm{O}(\mathrm{g})+\mathrm{OH}(\mathrm{g})$ and $T_{\text {gas }} \leq 35 \mathrm{~K}$. The abundances of the $\mathrm{CO}$ isotopologs ${ }^{13} \mathrm{CO}$ and $\mathrm{C}^{18} \mathrm{O}$ are calculated by scaling their abundances by factor $X_{\mathrm{CO}}^{\text {new }} / X_{\mathrm{CO}}^{\text {old }}$, that is, by how much the ${ }^{12} \mathrm{CO}$ abundance has decreased.

Figure 3 shows the effects of $\mathrm{CO}$ conversion through grainsurface chemistry on the CO abundances at 1,3, and 10 Myr for one of our disk models. As discussed above, the $\mathrm{CO}$ abundances are only recalculated below the dashed black lines. At $1 \mathrm{Myr}$ the $\mathrm{CO}$ abundance is still $\mathrm{CO} / \mathrm{H}_{2} \sim 10^{-4}$ and $\mathrm{CO}$ has not been depleted significantly. By $3 \mathrm{Myr}$ the $\mathrm{CO}$ abundance has dropped to $\mathrm{CO} / \mathrm{H}_{2} \sim 5 \times 10^{-5}$ higher up in the disk and $\mathrm{CO} / \mathrm{H}_{2} \sim 5 \times 10^{-6}$ close to the midplane. For ${ }^{13} \mathrm{CO}$ the effect of isotope-selective photodissociation is partly offset by the reaction ${ }^{13} \mathrm{C}^{+}+{ }^{12} \mathrm{CO} \rightarrow$ ${ }^{13} \mathrm{CO}+{ }^{12} \mathrm{C}^{+}+35 \mathrm{~K}$, which is energetically favored toward the production of ${ }^{13} \mathrm{CO}$ at low temperatures (see, e.g., Visser et al. 2009; Miotello et al.2014 and references therein).

Having a lower abundance and lacking a back reaction similar to ${ }^{13} \mathrm{CO}$ (see eq. 2 in Miotello et al. 2014), $\mathrm{C}^{18} \mathrm{O}$ is much more affected by isotope-selective photodissociation. As a result, $\mathrm{C}^{18} \mathrm{O}$ is confined to a layer deeper in the disk. This can be seen in Figure 3 , with the ${ }^{13} \mathrm{CO} 3-2$ emitting region lying higher up in the disk, fully above the black contour. The $\mathrm{C}^{18} \mathrm{O} 3-2$ emitting region lies closer toward the midplane and crosses the black contour. We can therefore expect the ${ }^{13} \mathrm{CO} 3-2$ flux not to be affected by the chemical conversion of $\mathrm{CO}$, whereas the $\mathrm{C}^{18} \mathrm{O} 3-2$ flux will be affected. After $10 \mathrm{Myr}$ almost all of the available $\mathrm{CO}$ has been converted, with $\mathrm{CO} / \mathrm{H}_{2} \lesssim 10^{-6}$ for the region where $\mathrm{CO}$ can be converted through cosmic-ray-driven chemistry. The ${ }^{13} \mathrm{CO}$ and $\mathrm{C}^{18} \mathrm{O}$ emitting regions have moved inward and upward to regions of the disk that still have a high $\mathrm{CO}$ abundance.

In this work we raytrace the ${ }^{13} \mathrm{CO}$ and $\mathrm{C}^{18} \mathrm{O} J=3-2$ lines, but tests show that the $J=2-1$ lines show the same qualitative behavior (cf. Appendices $\mathrm{B}$ and C). As a rule of thumb if the $J=3-2$ lines are optically thick, meaning that at early ages, when the disk is most massive, the ${ }^{13} \mathrm{CO} 2-1$ line is up to $5 \%$ fainter (in $\mathrm{Jy} \mathrm{km} \mathrm{s}^{-1}$ ) and the $\mathrm{C}^{18} \mathrm{O} 2-1$ line is up to $15 \%$ fainter. If the $J=3-2$ lines are optically thin, the $J=2-1$ fluxes are up to $30 \%$ lower for both ${ }^{13} \mathrm{CO}$ and $\mathrm{C}^{18} \mathrm{O}$.

\section{Results}

\subsection{CO isotopolog line fluxes: A viscously evolving disk}

As the disk evolves viscously, the characteristic radius increases and the disk spreads out. At the same time the disk mass decreases as material is accreted onto the star. The left panel of Figure 4 shows the surface density profile of the model with $M_{*}=0.32 \mathrm{M}_{\odot}$ and $\alpha_{\text {visc }}=10^{-3}$, corresponding to an initial disk mass $M_{\text {disk }}(t=0)=10^{-2} \mathrm{M}_{\odot}$, as it changes over time.

The middle panel of Figure 4 shows the corresponding ${ }^{13} \mathrm{CO}$ $J=3-2$ azimuthally averaged intensity profile. Throughout most of the disk the ${ }^{13} \mathrm{CO}$ emission is optically thick and decreases slowly with radius. In the outer part of the disk, at a radius between $\sim 100-400 \mathrm{AU}$ depending on the age of the model, the ${ }^{13} \mathrm{CO}$ emission drops off rapidly. Comparing to the left panel in Figure 4, this radius corresponds to a surface density of $\Sigma_{\text {gas }} \approx 10^{-3} \mathrm{~g} \mathrm{~cm}^{-2}$. Below this density, the ${ }^{13} \mathrm{CO}$ column is no longer high enough to effectively self-shield and ${ }^{13} \mathrm{CO}$ is rapidly removed from the disk beyond this radius.

As the ${ }^{13} \mathrm{CO}$ emission is optically thick throughout almost all of the disk, the integrated ${ }^{13} \mathrm{CO}$ flux is expected to increase over time as viscous expansion of the disk increases its emitting area. This is indeed what is seen in Figure 5, where the top panel shows the integrated ${ }^{13} \mathrm{CO} 3-2$ flux as a function of disk age. ${ }^{13} \mathrm{CO}$ intensity profiles of all of our models, shown in Appendix $\mathrm{A}$, indicate that the ${ }^{13} \mathrm{CO}$ emission is optically thick throughout the disk for all disk models with $\alpha_{\text {visc }} \leq 10^{-3}$. Overall, the ${ }^{13} \mathrm{CO}$ flux is therefore more of a disk size tracer than a disk mass tracer.

The rightmost panel of Figure 4 shows the time evolution of the $\mathrm{C}^{18} \mathrm{O}$ intensity profile for the disk model with $M_{*}=0.32 \mathrm{M}_{\odot}$, $\alpha_{\text {visc }}=10^{-3}$. The profile consists of two components: the inner $\sim 90 \mathrm{AU}$ is the emission of the bulk of the $\mathrm{C}^{18} \mathrm{O}$ in the disk, located just above the midplane. This emission is optically thick up to 3-5 Myr, after which the emission starts to become optically thin. The second component, visible beyond $100 \mathrm{AU}$, is two orders of magnitude fainter than the first component. This emission is optically thin and originates from a lower abundance warm finger of $\mathrm{C}^{18} \mathrm{O}$ located higher up in the disk (see Figure 2 in Miotello et al.2014).

While the first component of the $\mathrm{C}^{18} \mathrm{O}$ emission is optically thick, the total $\mathrm{C}^{18} \mathrm{O}$ increases with age as can be seen in the bottom panel of Figure 5. The total $\mathrm{C}^{18} \mathrm{O} 3-2$ flux increases up to $\sim 3 \mathrm{Myr}$. At this point the $\mathrm{C}^{18} \mathrm{O}$ emission within $\sim 100 \mathrm{AU}$ starts to become optically thin and the $\mathrm{C}^{18} \mathrm{O}$ integrated flux becomes a tracer of the disk mass instead of the disk size.

Whether the $\mathrm{C}^{18} \mathrm{O}$ integrated flux is predominantly optically thick and tracing the disk size or optically thin and tracing the disk mass is determined, to first order, by the mass and the size of the disk. For disk ages $\geq 1 \mathrm{Myr}$ the $\mathrm{C}^{18} \mathrm{O}$ emission of our models is optically thin throughout the disk if $M_{*} \leq 0.2 \mathrm{M}_{\odot}$ and $\alpha_{\text {visc }} \geq 10^{-3}\left(M_{\text {disk }}(t=1 \mathrm{Myr}) \leq 10^{-3} \mathrm{M}_{\odot}\right)$. However, higher mass disks $\left(M_{\text {disk }}(t=1 \mathrm{Myr})\right.$ around higher mass stars $\left(M_{*} \geq\right.$ $\left.0.32 \mathrm{M}_{\odot}\right)$ or disks that have low turbulence $\left(\alpha_{\text {visc }} \leq 10^{-4}\right)$ instead have optically thick $\mathrm{C}^{18} \mathrm{O} 3-2$ emission throughout most of the disk.

\subsection{CO isotopolog line fluxes: Effects of grain-surface chemistry}

Before we compare our models to the observations, we first look at the effects of conversion of $\mathrm{CO}$ through grain-surface chemistry on $\mathrm{CO}$ isotopolog line fluxes. As lowering the $\mathrm{CO}$ abundance decreases the ${ }^{13} \mathrm{CO}$ and $\mathrm{C}^{18} \mathrm{O}$ column densities, we expect the ${ }^{13} \mathrm{CO}$ and $\mathrm{C}^{18} \mathrm{O}$ emission to become optically thin at an earlier disk age.

Figure 6 shows $\mathrm{C}^{18} \mathrm{O}$ and ${ }^{13} \mathrm{CO} J=3-2$ integrated line fluxes for models with $M_{*}=0.32 \mathrm{M}_{\odot}$ and $\alpha_{\text {visc }}=\left[10^{-3}, 10^{-4}\right]$. The line fluxes without grain-surface chemistry, presented earlier in Figure 5, are included as dashed lines. Up to $1 \mathrm{Myr}$ there is no discernible difference between the models with and without grain-surface chemistry, as it takes time for the chemistry to lower the gas-phase $\mathrm{CO}$ abundance. We note here that the timescale for the grain-surface chemistry depends directly on the assumed cosmic-ray ionization rate $\zeta_{\mathrm{cr}}$, which we have assumed 

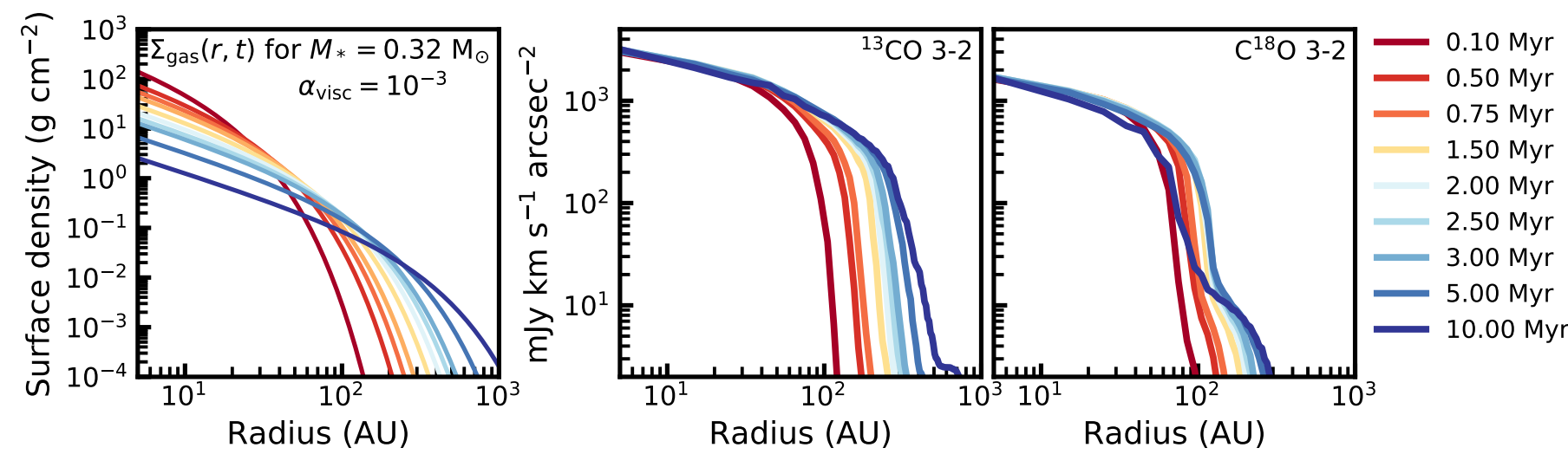

Fig. 4: Time evolution of the surface density profile (left panel), with the middle and right panel showing the corresponding ${ }^{13} \mathrm{CO}$ $J=3-2$ and $\mathrm{C}^{18} \mathrm{O} J=3-2$ intensity profiles, respectively. The model shown here has $M_{*}=0.32 \mathrm{M}_{\odot}, \alpha=10^{-3}$ and $M_{\text {disk }}(t=$ $0)=10^{-2} \mathrm{M}_{\odot}$. The colors, going from red to blue, show different time steps. ${ }^{13} \mathrm{CO}$ and $\mathrm{C}^{18} \mathrm{O} 3-2$ intensity profiles for all models can be found in Appendix A

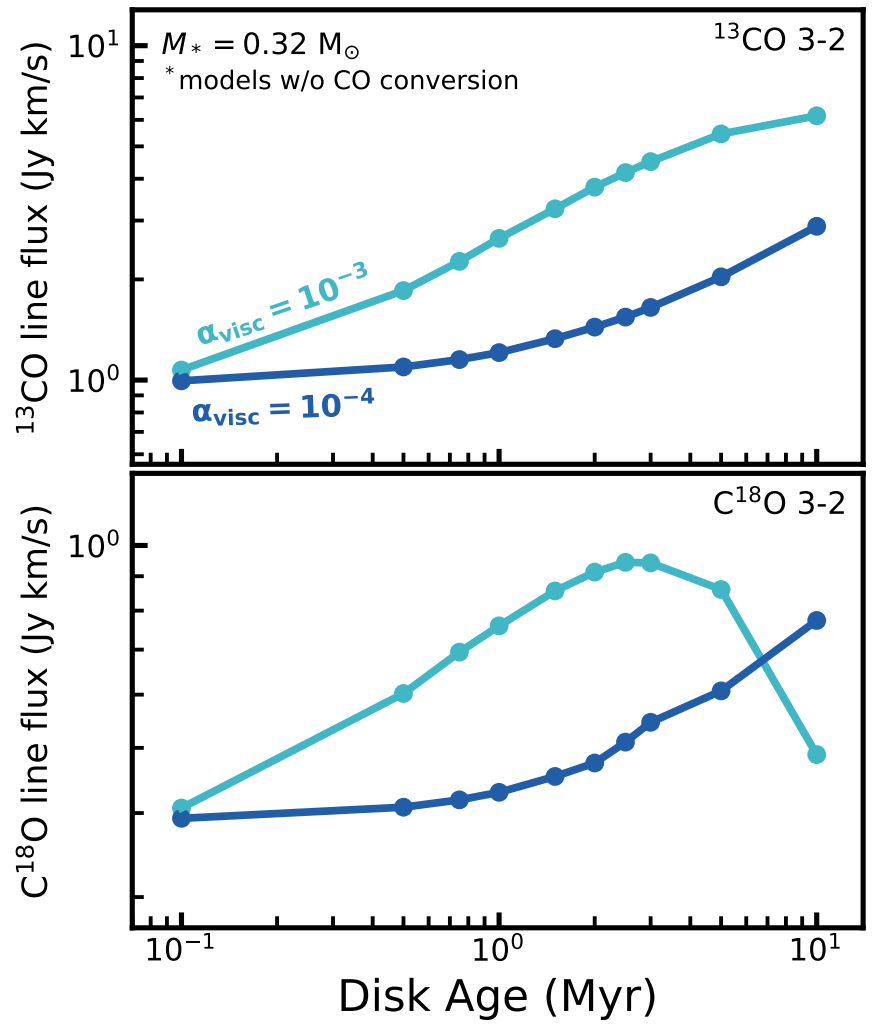

Fig. 5: ${ }^{13} \mathrm{CO}$ and $\mathrm{C}^{18} \mathrm{O} J=3-2$ line fluxes of a viscously evolving disk, shown in the top and bottom panel, respectively. Presented here are models with $M_{*}=0.32 \mathrm{M}_{\odot}, \alpha_{\text {visc }}=10^{-3}-$ $10^{-4}$ that have an initial disk mass $M_{\text {disk }}(t=0)=2 \times 10^{-3}-2 \times$ $10^{-2} \mathrm{M}_{\odot}$. We note that for these models $\mathrm{CO}$ conversion through grain-surface chemistry is not included.

to be $\zeta_{\text {cr }}=1 \times 10^{-17} \mathrm{~s}^{-1}$. For a higher $\zeta_{\text {cr }}$ the conversion of $\mathrm{CO}$ into other species will occur on a shorter timescale (see also Section 3.4.

For ${ }^{13} \mathrm{CO}$ there is almost no difference when grain-surface chemistry is included even after $1 \mathrm{Myr}$. As can be seen in Figure 3 the ${ }^{13} \mathrm{CO} 3-2$ emitting region lies almost completely above the

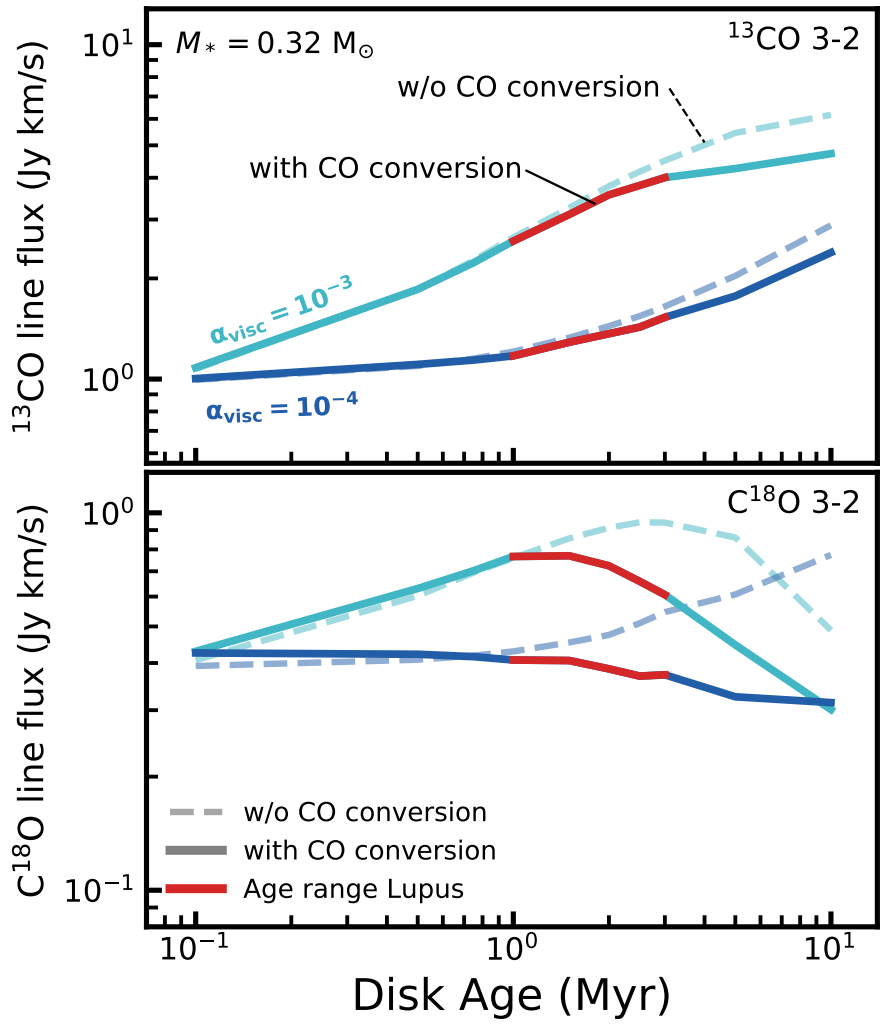

Fig. 6: ${ }^{13} \mathrm{CO}$ and $\mathrm{C}^{18} \mathrm{O} J=3-2$ line fluxes of a viscously evolving disk, shown in the top and bottom panel, respectively. Presented here are models with $M_{*}=0.32 \mathrm{M}_{\odot}, \alpha_{\text {visc }}=10^{-3}-10^{-4}$ that have an initial disk mass $M_{\text {disk }}(t=0)=2 \times 10^{-3}-2 \times$ $10^{-2} \mathrm{M}_{\odot}$. A full overview of the model fluxes can be found in Figure B.1. Solid lines show fluxes for models that include $\mathrm{CO}$ conversion through grain-surface chemistry. For comparison, dashed lines show fluxes for models without $\mathrm{CO}$ conversion as in Figure 5. The red line highlights the age range of disks in the Lupus star-forming region.

region of the disk where $\mathrm{CO}$ can be transformed on the grains. Grain-surface chemistry has a larger effect on the $\mathrm{C}^{18} \mathrm{O}$ fluxes 
because the $\mathrm{C}^{18} \mathrm{O} 3-2$ emitting region lies closer to the midplane, where the conversion of $\mathrm{CO}$ is most efficient (see, e.g., Figure 3). For both $\alpha_{\text {visc }}=10^{-3}$ and $10^{-4}$ the $\mathrm{C}^{18} \mathrm{O} 3-2$ flux starts to decrease at a disk age of $\sim 1.5 \mathrm{Myr}$. This indicates that the $\mathrm{C}^{18} \mathrm{O}$ emission is optically thin for a much larger disk masses (up to $M_{\text {disk }} \sim 2 \times 10^{-2} \mathrm{M}_{\odot}$, cf. Figure 1). At $10 \mathrm{Myr}$ grain-surface chemistry has lowered the $\mathrm{C}^{18} \mathrm{O} 3-2$ fluxes by a factor of $\sim 2-3$ compared to the model without $\mathrm{CO}$ conversion.

\subsection{Comparing to the Lupus disk population}

Recently Ansdell et al. (2016) have carried out an ALMA survey of the protoplanetary disk population in the Lupus star-forming region, in both continuum and ${ }^{12} \mathrm{CO},{ }^{13} \mathrm{CO}$ and $\mathrm{C}^{18} \mathrm{O}$ line emission (see also Ansdell et al. 2018). We examine whether the low ${ }^{13} \mathrm{CO}$ and $\mathrm{C}^{18} \mathrm{O} 3-2$ fluxes are compatible with viscous evolution.

Figure 7 shows the $\mathrm{C}^{18} \mathrm{O}$ and ${ }^{13} \mathrm{CO} J=3-2$ integrated line fluxes of our models and observations of protoplanetary disks in Lupus (Ansdell et al. 2016, Yen et al. 2018). To have a useful comparison, we have aligned the models and observations based on total (estimated) disk mass. For the observations we use $100 \times$ $M_{\text {dust }}$ as a proxy for the disk mass. This is equivalent to assuming that disks have a gas-to-dust mass ratio $\Delta_{\text {gd }}=100$, the canonical value for the ISM. It is likely that radial drift of larger grains and their subsequent accretion onto the star causes disks to have a $\Delta_{\mathrm{gd}}>100$. This would move the observations to the right in Figure 7. We note, however, that substructures detected in the continuum (e.g., ALMA Partnership et al. 2015; Andrews et al. 2016; Andrews et al. 2018, van Terwisga et al. 2018; Long et al. 2018, 2019; Cieza et al. 2020) could halt the inward drift of large grains (see e.g., Pinilla et al.|2012; Dullemond et al.|2018). This is discussed in more detail in Section 4.2 In our analysis we exclude transition disks with a resolved inner cavity in the dust continuum as our models do no represent their disk structure (see van der Marel et al.2018).

At the high mass end $\left(M_{\text {disk }} \gtrsim 5 \times 10^{-3} \mathrm{M}_{\odot}\right){ }^{13} \mathrm{CO} 3-2$ is detected for all disks and $\mathrm{C}^{18} \mathrm{O}$ 3-2 is detected for most disks in Lupus. The observed range of ${ }^{13} \mathrm{CO}$ fluxes is reasonably well reproduced by our models, although for individual objects the flux for the model with the corresponding mass might be a factor of two to four times higher. There are two disks, IM Lup (Sz 82) and HK Lup (Sz 98) that are either significantly brighter or fainter than our models. These two disks are among the largest disks in Lupus. Interestingly, while these two disks are of similar size and dust mass, they differ in ${ }^{13} \mathrm{CO} 3-2$ flux by more than an order of magnitude, suggesting that the processes that affect the abundance of CO can be very different in two very similarly looking disks. We note that our models are aimed at reproducing the average protoplanetary disk and we do not expect them to reproduce outliers. In a similar study Trapman et al. (2020) showed that a larger initial disk size of $30-50 \mathrm{AU}$ is required to reproduce the gas disk size of these large disks.

The $\mathrm{C}^{18} \mathrm{O}$ 3-2 fluxes detected for the high mass disks are reproduced by the models within a factor of $\sim 2$ for most disks. Four of the massive disks in Lupus, slightly less than half of the disks in this mass range, are not detected in $\mathrm{C}^{18} \mathrm{O} 3-2$. Our models overproduce these $\mathrm{C}^{18} \mathrm{O}$ upper limits by a factor of $\sim$ $2-4$, suggesting they either have a $\Delta_{\text {gd }} \ll 100$ or that they have lower $\mathrm{C}^{18} \mathrm{O}$ abundances than our models. The $\mathrm{C}^{18} \mathrm{O}$ fluxes in our models can be reduced by increasing the cosmic-ray ionization rate (see Section 3.4 and Figure D.1).

For lower disk masses $\left(5 \times 10^{-4} \mathrm{M}_{\odot} \lesssim M_{\text {disk }} \lesssim 5 \times 10^{-3} \mathrm{M}_{\odot}\right)$ the majority of disks in Lupus are still detected in ${ }^{13} \mathrm{CO}$ but only a few are also detected in $\mathrm{C}^{18} \mathrm{O}$. Here the ${ }^{13} \mathrm{CO}$ fluxes of our models and the observations start to diverge. While the brightest observed ${ }^{13} \mathrm{CO}$ fluxes are still reproduced by the models, there is up to an order of magnitude difference between the model ${ }^{13} \mathrm{CO}$ fluxes and the bulk of the ${ }^{13} \mathrm{CO}$ detections and the ${ }^{13} \mathrm{CO}$ upper limits.

For $\mathrm{C}^{18} \mathrm{O}$, comparing the models to the observations becomes more difficult due to the low number of $\mathrm{C}^{18} \mathrm{O} 3-2$ detections in Lupus. The few $\mathrm{C}^{18} \mathrm{O}$ detections in this mass range are reproduced by the models. However, these are the same disks whose bright ${ }^{13} \mathrm{CO}$ emission is also reproduced by the models. The $\mathrm{C}^{18} \mathrm{O}$ fluxes of the models do get low enough to match the observed $\mathrm{C}^{18} \mathrm{O}$ upper limits $\left(\sim 0.1 \mathrm{Jy} \mathrm{km} \mathrm{s}^{-1}\right)$, but only when the disk models are $\sim 10$ Myr old. This is much older than most disks in Lupus, which are estimated to be 1-3 Myr old.

Ansdell et al. (2016) stacked the disks that were detected in the continuum but not in ${ }^{13} \mathrm{CO} \mathrm{3-2}$ and $\mathrm{C}^{18} \mathrm{O} 3-2$. First, stacking the 25 sources that were detected in the continuum and ${ }^{13} \mathrm{CO}$ resulted in a mean continuum flux of $70 \mathrm{mJy}$, corresponding to a dust mass of $\sim 5 \times 10^{-5} \mathrm{M}_{\odot}$, with a detected mean $\mathrm{C}^{18} \mathrm{O}$ 3-2 flux of $206 \pm 31 \mathrm{mJy} \mathrm{km} \mathrm{s}^{-1}$. Stacking the 26 sources detected in the continuum but not detected in both ${ }^{13} \mathrm{CO} 3-2$ and $\mathrm{C}^{18} \mathrm{O} 3-2$ provided a much deeper mean $\mathrm{C}^{18} \mathrm{O} 3-2$ upper limit of $42 \mathrm{mJy} \mathrm{km} \mathrm{s}^{-1}$. We note that we have scaled the fluxes to a distance of $160 \mathrm{pc}$, the average distance to the Lupus clouds based on Gaia DR2 measurements (Brown et al. 2018, Bailer-Jones et al. 2018), instead of the 200 pc used by Ansdell et al. (2016). This stacked upper limit lies a factor of five to ten lower than our model fluxes, similar to what was found for ${ }^{13} \mathrm{CO}$, suggesting that both ${ }^{13} \mathrm{CO} 3-2$ and $\mathrm{C}^{18} \mathrm{O} 3-2$ fluxes are overproduced by our models, even if chemical conversion of $\mathrm{CO}$ is included.

For disks with $M_{\text {disk }}=100 \times M_{\text {dust }} \lesssim 5 \times 10^{-4} \mathrm{M}_{\odot}$ only a handful of disks are detected in ${ }^{13} \mathrm{CO}$ and none are detected in $\mathrm{C}^{18} \mathrm{O}$. While the ${ }^{13} \mathrm{CO}$ fluxes of the models have decreased for these lower gas masses, the model fluxes are still at least a factor of five higher than the observed ${ }^{13} \mathrm{CO}$ upper limits. In comparison the $\mathrm{C}^{18} \mathrm{O}$ fluxes of the model have decreased with $M_{\text {disk }}$ and are consistent with the observed $\mathrm{C}^{18} \mathrm{O}$ upper limits.

To summarize, our viscously evolving disk models that include $\mathrm{CO}$ conversion are able to explain most observed ${ }^{13} \mathrm{CO}$ and $\mathrm{C}^{18} \mathrm{O}$ fluxes for high mass $\operatorname{disks}\left(M_{\text {disk }} \gtrsim 5 \times 10^{-5} \mathrm{M}_{\odot}\right)$ in Lupus. For lower mass disks the model ${ }^{13} \mathrm{CO}$ and $\mathrm{C}^{18} \mathrm{O}$ fluxes are up to an order of magnitude higher than what has been observed.

\subsection{Cosmic-ray ionization rate required to match observed $\mathrm{CO}$ isotopolog fluxes}

The previous Section showed that for disk masses below $5 \times 10^{-3} \mathrm{M}_{\odot}$ the model ${ }^{13} \mathrm{CO}$ and $\mathrm{C}^{18} \mathrm{O}$ fluxes are a factor of five to ten times higher than what is observed in Lupus. The cosmicray ionization rate $\zeta_{\mathrm{cr}}$ is one of the main factors that determines the rate at which $\mathrm{CO}$ is converted into $\mathrm{CO}_{2}, \mathrm{CH}_{4}$ and $\mathrm{CH}_{3} \mathrm{OH}$. In our models we have assumed a moderate $\zeta_{\mathrm{cr}} \sim 10^{-17} \mathrm{~s}^{-1}$, comparable to the rate found in dense molecular clouds (see, e.g., Black et al. 1990). The cosmic-ray ionization rate in disks is highly uncertain, so it is possible that $\zeta_{\mathrm{cr}}$ is higher, especially if activity from the young star at the center of the disk is contributing (see, e.g., Rab et al. 2017; Padovani et al. 2018). However, it should be noted that there is also evidence for a much lower cosmic-ray ionization rate in disks $\left(\zeta_{\mathrm{cr}} \sim 10^{-19}-10^{-18} \mathrm{~s}^{-1}\right)$ as a result of the disk being shielded by the stellar magnetic field and disk winds (Cleeves et al. 2013, Cleeves et al. 2015). However, as reducing $\zeta_{\text {cr }}$ only decreases the effectiveness of the chemical conversion of $\mathrm{CO}$ we do not examine it here. Instead we exam- 

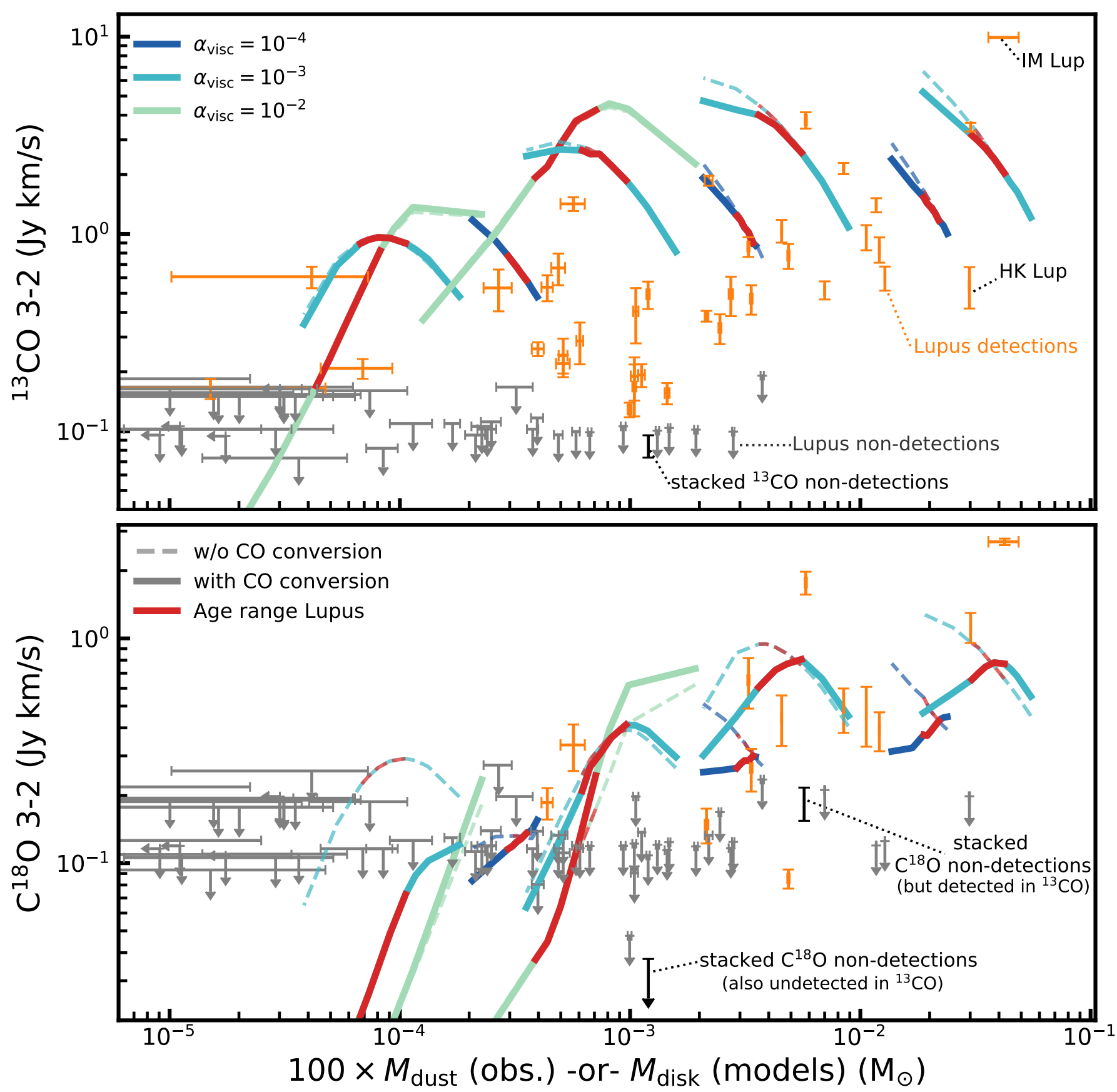

Fig. 7: ${ }^{13} \mathrm{CO}$ (top) and $\mathrm{C}^{18} \mathrm{O}$ (bottom) $J=3-2$ line fluxes versus disk mass. Solid lines show models that include $\mathrm{CO}$ conversion through grain-surface chemistry. For comparison, dashed lines show the models without grain-surface chemistry. For the observations, shown in orange, we use $M_{\text {gas }} \simeq 100 \times M_{\text {dust }}$ as a proxy for the disk mass (see, e.g., Ansdell et al. 2016, Ansdell et al. 2018, Yen et al. 2018). Colors show models with different viscous alpha. Models with age between 1 and 3 Myr, the approximate age of Lupus, are highlighted in red. We note that in our models $M_{\text {disk }}$ decreases with time, meaning that time runs right to left in this figure for our models. Observations for which we only have an upper limit on the ${ }^{13} \mathrm{CO}$ or $\mathrm{C}^{18} \mathrm{O}$ line flux are shown in gray. Stacked non-detections are shown in black (cf. Section 3.3).

ine if increasing $\zeta_{\text {cr }}$ would allow us to reproduce the observations and what $\zeta_{\text {cr }}$ would be needed to do so in a 1-3 Myr time period. We focus on the mass range between $M_{\text {disk }}=5 \times 10^{-4} \mathrm{M}_{\odot}$ and $M_{\text {disk }}=10^{-2} \mathrm{M}_{\odot}$ where the difference in fluxes between the models and the observations is the largest. Similarly, we focus on models with $\alpha_{\text {visc }}=10^{-3}$ and $M_{*}=[0.2,0.32] \mathrm{M}_{\odot}$, which span a similar disk mass range.
Figure 8 shows the ${ }^{13} \mathrm{CO}$ and $\mathrm{C}^{18} \mathrm{O} 3-2$ fluxes after $\mathrm{CO}$ abundances were recalculated using a higher $\zeta_{\mathrm{cr}}$ than is used in the models presented previously $\left(\zeta_{\mathrm{cr}}=1 \times 10^{-17} \mathrm{~s}^{-1}\right.$; see Table 2.) The ${ }^{13} \mathrm{CO} 3-2$ emission is only weakly dependent on the cosmicray ionization rate, with less than $\sim 10 \%$ differences in flux. Again, this is due to the ${ }^{13} \mathrm{CO}$ emitting region $(z / r \sim 0.2-0.35)$ being higher than the region of CO removal $(z / r \lesssim 0.15)$ In Sec- 


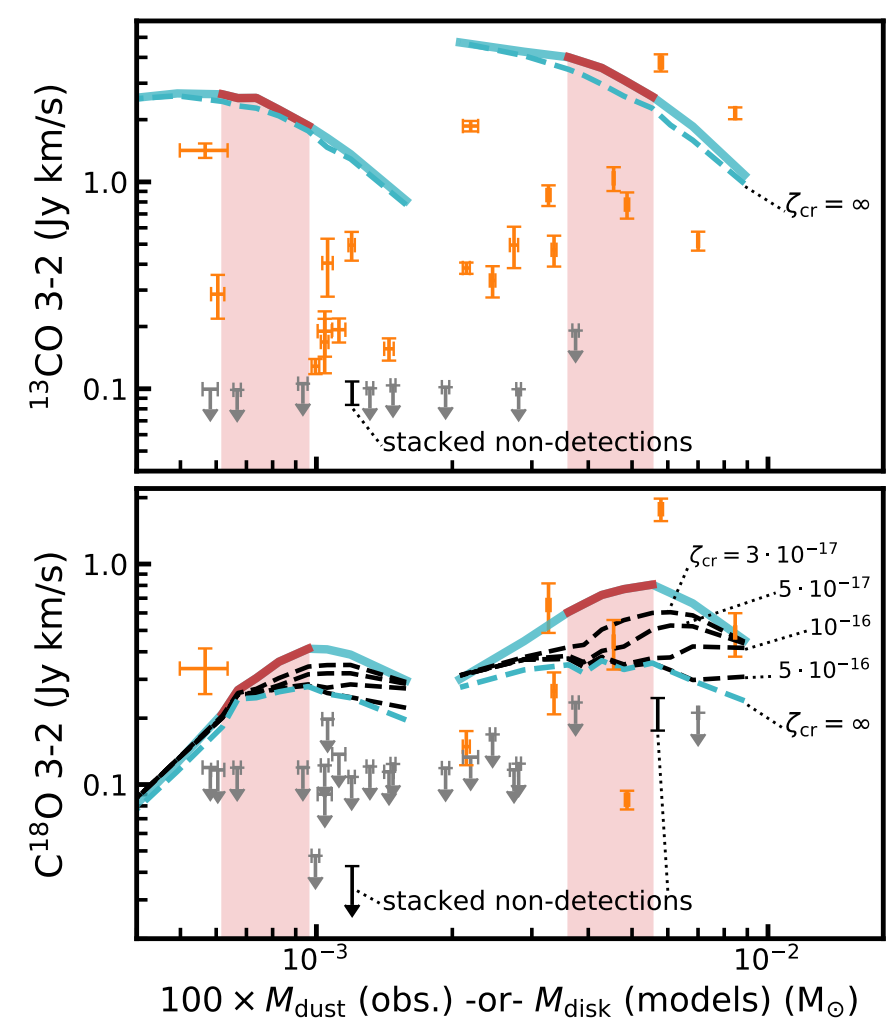

Fig. 8: Effect of the cosmic ray ionization rate on ${ }^{13} \mathrm{CO}$ and $\mathrm{C}^{18} \mathrm{O} J=3-2$ line fluxes, shown in the top and bottom panel respectively. Models shown here have $\alpha_{\text {visc }}=10^{-3}$ and $M_{*}=[0.2,0.32] \mathrm{M}_{\odot}$. Solid light blue lines show the model line fluxes where $\mathrm{CO}$ conversion through grain-surface chemistry is calculated with a cosmic ray ionization rate $\zeta_{\mathrm{cr}}=1 \times 10^{-17} \mathrm{~s}^{-1}$. For the black dashed lines $\zeta_{\mathrm{cr}}$ is increased up to $5 \times 10^{-16} \mathrm{~s}^{-1}$. The dashed blue line shows the upper limit of the effect of grainsurface chemistry, where we have removed all CO in the region of the disk where $\mathrm{CO}$ conversion by grain-surface chemistry is effective. Observations in Lupus are shown in orange if detected and in gray if an upper limit (Ansdell et al. 2016; Yen et al. 2018). The stacked non-detections are shown in black (see also Section 3.3). The red shaded lane highlights the age range of the Lupus star-forming region.

tion 4.1 we discuss alternative ways to reconcile the ${ }^{13} \mathrm{CO} 3-2$ observations with our models.

The bottom panel of Figure 8 shows that, in contrast to ${ }^{13} \mathrm{CO}$, increasing $\zeta_{\text {cr }}$ has a larger effect on the $\mathrm{C}^{18} \mathrm{O}$ fluxes, but not enough. Increasing $\zeta_{\text {cr }}$ from $1 \times 10^{-17} \mathrm{~s}^{-1}$ to $5 \times 10^{-17}-10^{-16} \mathrm{~s}^{-1}$ is sufficient to explain almost all of the $\mathrm{C}^{18} \mathrm{O}$ detections for disks with $100 \times M_{\text {dust }} \gtrsim 2 \times 10^{-3} M_{\odot}$. However, the figure also shows that by increasing $\zeta_{\text {cr }}$ the $\mathrm{C}^{18} \mathrm{O} 3-2$ fluxes can reduced to at most $\sim 0.2 \mathrm{Jy} \mathrm{km} \mathrm{s}^{-1}$, which is still a factor of $\sim 2$ higher than the observed $\mathrm{C}^{18} \mathrm{O}$ upper limits in Lupus. Moreover, the fluxes obtained from our models remain a factor of $\sim 5$ higher than the stacked $\mathrm{C}^{18} \mathrm{O}$ 3-2 upper limits. This indicates that increasing the amount of cosmic-ray ionization in our models by itself cannot explain the faintest $\mathrm{C}^{18} \mathrm{O}$ 3-2 fluxes.

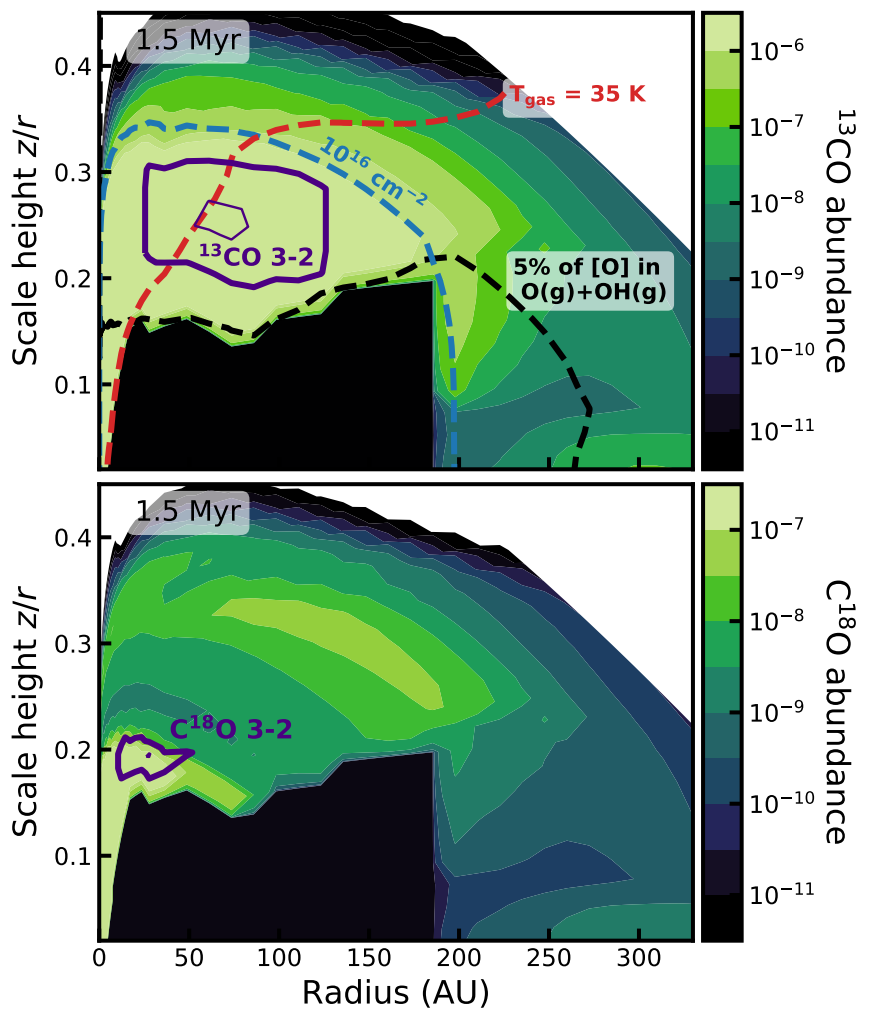

Fig. 9: ${ }^{13} \mathrm{CO}$ (top) and $\mathrm{C}^{18} \mathrm{O}$ (bottom) abundance structure (colors) and $J=3-2$ emitting regions (purple contours) for an example disk model $\left(M_{*}=0.32 \mathrm{M}_{\odot}, \alpha_{\text {visc }}=10^{-3}\right.$ and a disk age of $1.5 \mathrm{Myr}$ ). The contours shown are the same as in Figure 2

\section{Discussion}

\subsection{Reproducing ${ }^{13} \mathrm{CO}$ 3-2 line fluxes observed in Lupus}

In Section 3.3 it was found that our models overproduce the ${ }^{13} \mathrm{CO} 3-2$ and $\mathrm{C}^{18} \mathrm{O} 3-2$ observation in Lupus by a factor of five to ten for disks with $M_{\text {disk }} \lesssim 5 \times 10^{-3} \mathrm{M}_{\odot}$. By increasing the cosmic-ray ionization rate from $\zeta_{\mathrm{cr}}=1 \times 10^{-17} \mathrm{~s}^{-1}$ to $10^{-16} \mathrm{~s}^{-1}$ it is possible to decrease the $\mathrm{C}^{18} \mathrm{O} 3-2$ fluxes of our models to within a factor of two of the observed upper limits. However, the ${ }^{13} \mathrm{CO} 3-2$ emission originates predominantly from a layer higher up in the disk where $\mathrm{CO}$ is not being efficiently converted into other species. As such, the ${ }^{13} \mathrm{CO} 3-2$ fluxes of our models remain a factor of $10-30$ times higher than the observations, even after removing all $\mathrm{CO}$ from region of the disk where $\mathrm{CO}$ grain-surface chemistry is effective. Here, we examine the emitting regions of ${ }^{13} \mathrm{CO} 3-2$ and $\mathrm{C}^{18} \mathrm{O}$ 3-2 and we discuss processes that could reduce the ${ }^{13} \mathrm{CO}$ and $\mathrm{C}^{18} \mathrm{O}$ fluxes to the point where they are in agreement with both detections and upper limits seen in observations.

Figure 9 shows an example of the ${ }^{13} \mathrm{CO}$ and $\mathrm{C}^{18} \mathrm{O}$ emitting regions in a model with maximum $\mathrm{CO}$ conversion. The ${ }^{13} \mathrm{CO}$ emitting region is located high up in the disk $(z / r \sim 0.25-0.4)$, fully above the region where $\mathrm{CO}$ can be removed $(z / r \lesssim 0.15)$. The presence of oxygen in the gas phase in this layer indicates that it cannot be locked up efficiently in $\mathrm{H}_{2} \mathrm{O}$, meaning it is available to react with the carbon released from the destruction of $\mathrm{CO}$ to reform CO (see also Schwarz et al. 2018). It should be noted a small amount of carbon can go into other molecules such as $\mathrm{H}_{2} \mathrm{CO}$ (e.g., Loomis et al. 2015, Öberg et al. 2017, Terwisscha 
van Scheltinga et al. 2020) and $\mathrm{C}_{2} \mathrm{H}$ (e.g., Kastner et al. 2015; Bergin et al. 2016; Cleeves et al. 2018, Bergner et al. 2019, Miotello et al. (2019), but in this FUV dominated layer CO remains the main carbon carrier (see Figure 18 in Schwarz et al. 2018). The $\mathrm{C}^{18} \mathrm{O}$ emitting region, shown in the bottom panel of Figure 9 lies much deeper in the disk, at a height of $z / r \sim 0.18$. With efficient $\mathrm{CO}$ conversion the $\mathrm{C}^{18} \mathrm{O}$ emitting region is very compact and extends only slightly above the oxygen-threshold. Unlike for ${ }^{13} \mathrm{CO}$ it is therefore plausible that a slight change in for example the height of the disk will push the $\mathrm{C}^{18} \mathrm{O}$ abundant region below the oxygen-threshold, thus drastically reducing the observed $\mathrm{C}^{18} \mathrm{O}$ 3-2 fluxes. In the rest of this section we therefore focus on lowering the ${ }^{13} \mathrm{CO} 3-2$ fluxes, which are in stronger violation with the observations.

Knowing the location of the ${ }^{13} \mathrm{CO}$ emission, we can discuss processes by which the emission can be reduced.

A colder disk: Given that the ${ }^{13} \mathrm{CO} 3-2$ emission is optically thick, a lower disk temperature could potentially explain the low ${ }^{13} \mathrm{CO} 3-2$ emission in Lupus. Lowering $T_{\text {gas }}$ would decrease the brightness of the optically thick line. The temperature of a disk is predominantly set by its vertical structure. Our models all have the same height, $H=R h=h_{c}\left(R / R_{c}\right)^{\psi}=0.1\left(R / R_{c}\right)^{0.15}$ (see Section 2.3. In reality, however, it is unlikely that all disks have the same height. The vertical structure that is assumed in our disk models is thought to be a good representation of the average disk vertical structure, but there are only a few disks, biased toward high disk masses, for which the disk height has been estimated using the scattering surface observed in scattered light (see, e.g., Avenhaus et al. 2018, Garufi et al. 2020). It is therefore possible that lower mass disks are much flatter $\left(h_{c} \ll 0.1\right)$, and therefore colder, than previously thought.

A lower scale height would also increase the fraction of $\mathrm{CO}$ in the disk that can be converted. Reducing the scale height of the disk would concentrate the mass in the shielded midplane, where the cosmic-ray-driven conversion of $\mathrm{CO}$ is efficient.

A colder disk cannot explain all of the disks observed to be underabundant in CO. The TW Hya disk is thought to be underabundant in CO by a factor of 10-100 (see, e.g., Favre et al.2013; Du et al. 2015; Bergin et al. 2016, Kama et al. 2016, Trapman et al. 2017; McClure et al. 2020). This disk is also known to be much more flared $(\psi \sim 0.3)$, and therefore warmer, than the disk models presented here (see, e.g., Kama et al. 2016, van Boekel et al. 2017; Schwarz et al. 2016, Calahan et al.2020). Although anecdotal, the example of TW Hya shows that a colder disk cannot be the sole explanation for the low observed ${ }^{13} \mathrm{CO} 3-2$ fluxes (see also Fedele et al.2016).

Smaller disks: Another way to explain the low ${ }^{13} \mathrm{CO} 3-2$ fluxes would be that disks are smaller than our models. Trapman et al. (2020) showed that our models are consistent with observed gas disk sizes in Lupus, measured from the extent of the ${ }^{12} \mathrm{CO} J=2-1$ emission. However, these observations are biased toward the most massive disks around the most massive stars in the Lupus disk population. Comparing their results with the observations presented in Figure 7 reveals that there are only three disks below $M_{\text {disk }}=3 \times 10^{-3} \mathrm{M}_{\odot}$ for which the gas disk size has been measured: $\mathrm{Sz} 133$ (238 AU), Sz 65 (172 AU), and $\mathrm{Sz} 73$ (103 AU). Of these, only $\mathrm{Sz} 73$ has a ${ }^{13} \mathrm{CO}$ flux that lies a factor of $\sim 2$ below the predictions of our models. For the remaining disks of similar or lower mass the gas disk size is unknown. Without knowing their gas disk size, it could be possible that the low ${ }^{13} \mathrm{CO}$ fluxes can be explained by a compact gas disk.

The optically thick emitting region of our disk models with $M_{\text {disk }} \lesssim 5 \times 10^{-3} \mathrm{M}_{\odot}$ has a radius of $\sim 30-150 \mathrm{AU}$, depending on the mass and $R_{\mathrm{c}}$ of the disk model. The ${ }^{13} \mathrm{CO} 3-2$ flux scales

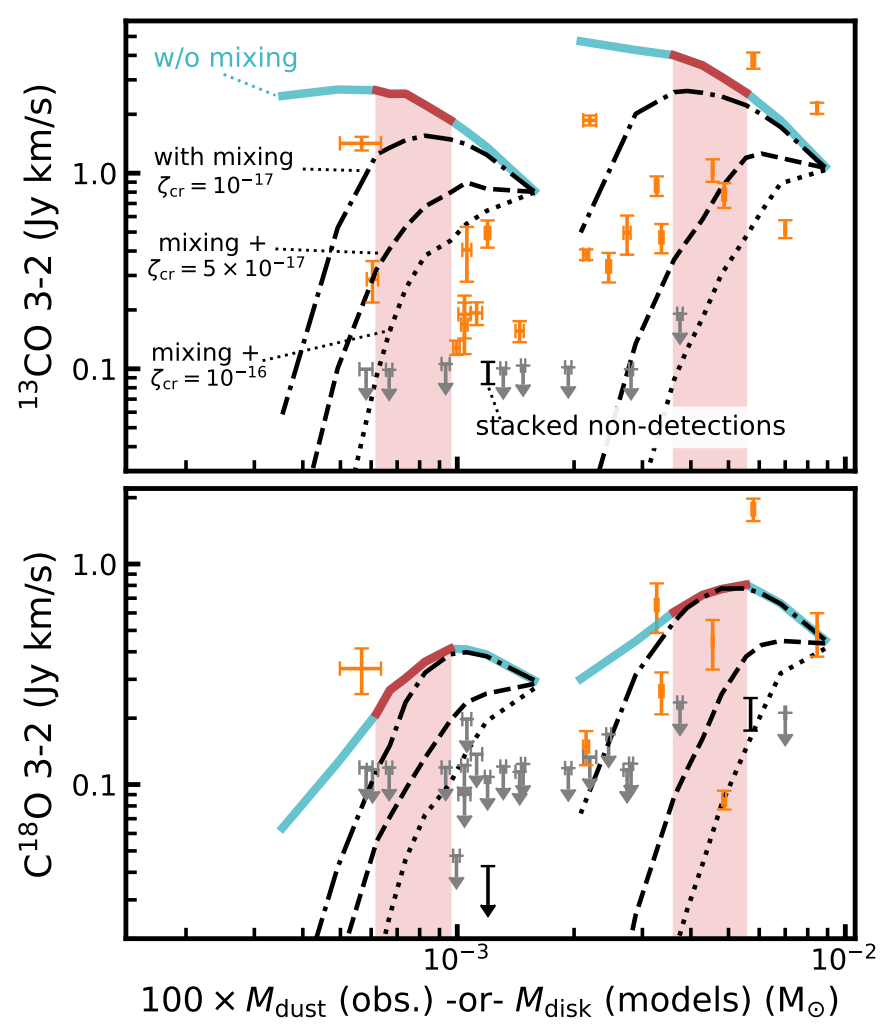

Fig. 10: ${ }^{13} \mathrm{CO} 3-2$ model fluxes after applying vertical mixing (dash-dotted black lines) compared the observation in Lupus (orange markers). Upper limits are shown in gray. The stacked nondetections are shown in black (see also Section 3.3). Shown here are disk models with $\alpha_{\text {visc }}=10^{-3}$ and $M_{*}=[0.2,0.32] \mathrm{M}_{\odot}$. For reference, the model fluxes without vertical mixing are shown in light blue. The dashed and dotted black lines show models where mixing is combined with higher cosmic-ray ionization rates (see also Section 3.4). The red shaded lane highlights the age range of the Lupus star-forming region.

with emitting area $\left(F \propto R^{2}\right)$. To reduce the ${ }^{13} \mathrm{CO} 3-2$ flux of these models by a factor of $10-30$ and bring them in line with the observations, the disk size has to be reduced by a factor of three to five, to $\sim 10-50 \mathrm{AU}$. We note that this is the disk size as measured from the ${ }^{13} \mathrm{CO} 3-2$ emission. Trapman et al. (2019) found that the gas disk size measured from ${ }^{12} \mathrm{CO}$ is $30-35 \%$ larger than the gas disk size measured from ${ }^{13} \mathrm{CO}$, assuming that both ${ }^{12} \mathrm{CO}$ and ${ }^{13} \mathrm{CO}$ are optically thick. This would suggest that disks with dust masses $\lesssim 3 \times 10^{-4} \mathrm{M}_{\odot}$ need to have gas disk sizes on the order of $\sim 40-70 \mathrm{AU}$ as measured from ${ }^{12} \mathrm{CO}$ if small disks are the explanation for the low ${ }^{13} \mathrm{CO}$ fluxes. It should be noted however that disks with similar ${ }^{13} \mathrm{CO}$ flux can have very different gas disk sizes (e.g., Sz 129 (140 AU) and HK Lup (358 AU)), suggesting that ${ }^{13} \mathrm{CO}$ might not always be optically thick and that compact disks might not be the sole explanation for the faint ${ }^{13} \mathrm{CO}$ emission seen in observations. Deeper high resolution $\left(\lesssim 0^{\prime \prime} .2\right){ }^{12} \mathrm{CO}$ and ${ }^{13} \mathrm{CO}$ observations of low mass disks are required to test this theory.

Vertical mixing: It is very likely that turbulence in protoplanetary disks mixes material both vertically and radially. Through vertical mixing the $\mathrm{CO}$-rich material higher up in the disk would be moved down toward the midplane, where the $\mathrm{CO}$ can then be converted into other species via grain-surface chemistry. If the mixing timescale is much shorter than the lifetime of the disk the 
$\mathrm{CO}$ will be well mixed and the $\mathrm{CO}$ abundance higher up in the disk will match that of the CO-poor material close to the midplane (see, e.g., Willacy et al. 2006; Semenov \& Wiebe 2011; Krijt et al. 2018, 2020).

Figure 10 shows the effect of vertical mixing on the ${ }^{13} \mathrm{CO}$ and $\mathrm{C}^{18} \mathrm{O}$ 3-2 fluxes. Here we have approximated the effect of mixing by setting the $\mathrm{CO}$ abundance higher up in the disk equal to the mass-weighted average $\mathrm{CO}$ abundance in the region where we compute the grain-surface chemistry. To obtain such efficient mixing requires a mixing timescale that is much shorter than the disk age. Based on turbulent diffusion, Krijt et al. (2020) calculate a vertical mixing timescale of $t_{z} \approx 2.5 \times 10^{3} \mathrm{yr}$ for one scale height at $30 \mathrm{AU}$ assuming a turbulent strength corresponding to $\alpha=10^{-4}$, which is also the lowest level of turbulence assumed in our models. We have also assumed that mixing goes up to the ${ }^{13} \mathrm{CO}$ emitting layer at $z / r \sim 0.25-0.4$.

Up to 3 Myr vertical mixing does not significantly affect the ${ }^{13} \mathrm{CO}$ and $\mathrm{C}^{18} \mathrm{O}$ fluxes for the standard case of $\zeta_{\mathrm{cr}}=1 \times 10^{-17} \mathrm{~s}^{-1}$. While the material in the upper layer of the disk is now well mixed with the material below, the grain-surface chemistry has not had enough time to decrease the mass-averaged $\mathrm{CO}$ abundance to the point where it has a noticeable effect on the isotopolog line fluxes. After $3 \mathrm{Myr}$ both the ${ }^{13} \mathrm{CO}$ flux has started to decrease rapidly and by $10 \mathrm{Myr}$ the fluxes from the our models are at or below the level of the observed upper limits in Lupus.

The effect of mixing on the ${ }^{13} \mathrm{CO}$ and $\mathrm{C}^{18} \mathrm{O} 3-2$ fluxes is limited to how quickly the chemistry is able to decrease the mass-averaged $\mathrm{CO}$ abundance. It is therefore interesting to examine whether a combination of vertical mixing and a higher cosmic-ray ionization rate $\zeta_{\text {cr }}$ can explain the ${ }^{13} \mathrm{CO} 3-2$ observations in Lupus within 1-3 Myr age of the region. We included vertical mixing in our models with $\zeta_{\mathrm{cr}}=5 \times 10^{-17}-10^{-16} \mathrm{~s}^{-1}$ and present the resulting fluxes in Figure 10 . With $\mathrm{CO}$ conversion now occurring at an increased rate, vertical mixing has a much larger impact at earlier disk ages. Within $3 \mathrm{Myr}$ the model fluxes have decreased to the same level as the faintest ${ }^{13} \mathrm{CO}$ and $\mathrm{C}^{18} \mathrm{O}$ detections and upper limits in Lupus. By combining vertical mixing with a high cosmic-ray ionization rate of $\zeta_{\mathrm{cr}}=5 \times 10^{-17}-10^{-16} \mathrm{~s}^{-1}$ we can explain all ${ }^{13} \mathrm{CO} 3-2$ and $\mathrm{C}^{18} \mathrm{O} 3-2$ observations in Lupus.

This is in agreement with recent results of Krijt et al. (2020), who investigated processes that have been invoked to explain low CO abundances inferred for disks, such as chemical conversion of $\mathrm{CO}$, turbulent diffusion of gas and small grains and locking up $\mathrm{CO}$ in large bodies. They show that a combination of vertical mixing and the chemical conversion of $\mathrm{CO}$ can decrease the $\mathrm{CO}$ abundance to $10^{-6}-10^{-5}$ up to $z / r \sim 0.2$, which is the upper edge of their model. This is similar to the mass-weighted average $\mathrm{CO}$ abundance of the viscous disk models that reproduce the observed ${ }^{13} \mathrm{CO}$ and $\mathrm{C}^{18} \mathrm{O} 3-2$ upper limits (see Figure 10. We should note however that to lower the ${ }^{13} \mathrm{CO}$ flux mixing must be efficient up to at least $z / r \sim 0.3$ (see Figure 9). Krijt et al. (2020) also show that including the locking up of $\mathrm{CO}$ ice into larger bodies in addition to the chemical conversion of $\mathrm{CO}$ can further decrease the $\mathrm{CO}$ abundance to below $10^{-6}$ at $z / r \sim 0.2$ for $\zeta_{\text {cr }}=1 \times 10^{-17} \mathrm{~s}^{-1}$. The combination of these processes could therefore potentially explain the low observed fluxes without having to invoke a high cosmic-ray ionization.

The effect of vertical mixing combined with the chemical conversion of $\mathrm{CO}$ is expected to lower the total volatile carbon abundance in the upper layers of the disk. Observations of atomic carbon in TW Hya indeed show evidence that the upper layer of the disk is carbon poor (see Kama et al. 2016), but there is insufficient observational evidence to show that this extends to all protoplanetary disks. Krijt et al. (2020) also find that vertical mixing and chemical conversion of $\mathrm{CO}$ leads to $(\mathrm{C} / \mathrm{O})_{\text {gas }}>1$ in the upper layer of the disk (see their Figure 10). This prediction is in agreement with the bright ring-shaped $\mathrm{C}_{2} \mathrm{H}$ emission that has been detected in a number of disks (see, e.g., Kastner et al. 2015, Bergin et al. 2016; Cleeves et al. 2018; Miotello et al. 2019; Bergner et al. 2019). However, it should be noted that it is still unclear how the $\mathrm{C}_{2} \mathrm{H}$ emission is related to the total amount of carbon that has been removed from the disk (see e.g., the discussion in Miotello et al. 2019).

\subsection{Alternative explanations}

Assumed gas-to-dust mass ratio: In Section 3.3 we compared our models to observations of disks in Lupus. In this comparison we used $M_{\text {disk }} \approx 100 \times M_{\text {dust }}$, which is equivalent to assuming that the disk has inherited the ISM gas-to-dust mass ratio of $\Delta_{\mathrm{gd}}=100$. However, most of the dust mass in protoplanetary is made up of large grains, which are expected to radially drift inward where they are accreted onto the star. This process would lead to $\Delta_{\mathrm{gd}} \gg 100$, with simulations of dust evolution in protoplanetary disks showing values as high as $\Delta_{\mathrm{gd}}=10^{3}-10^{4}$ (see, e.g., Birnstiel et al. 2012). Increasing the assumed gas-to-dust mass ratio would shift the observations to the right in Figure 7 . as it would increase the gas mass we associate with each of the sources. While this could change which models the observations are compared to, it would not significantly affect our results. A small increase $\left(\Delta_{\mathrm{gd}}=200-300\right)$ might help the comparison between the observations and our models at the high mass end. The main effect of increasing $\Delta_{\mathrm{gd}}$ would be increasing the gas mass threshold, currently $M_{\text {disk }} \lesssim 5 \times 10^{-3} \mathrm{M}_{\odot}$, below which we require a higher cosmic-ray ionization rate to match our models to the observations (see also Figure D.1).

We note here that lowering the gas-to-dust mass ratio of the observed sources to $\Delta_{\mathrm{gd}}=1-10$ would also allow us to reproduce the observed ${ }^{13} \mathrm{CO}$ and $\mathrm{C}^{18} \mathrm{O}$ fluxes with our models. This is in essence the same result as was obtained by Ansdell et al. (2016) and Miotello et al. (2017), who showed that gas masses derived from the ${ }^{15} \mathrm{CO}$ and $\mathrm{C}^{18} \mathrm{O}$ fluxes suggest that disks have low gas-to-dust mass ratios. However, these lower gas masses would no longer be consistent with observed stellar mass accretion rates under the assumption of viscous evolution (see Manara et al. 2016).

On the other side of this comparison are the initial disk masses used for our models. As outlined in Section 2.2, the initial disk mass $M_{\text {disk }}(t=0)$ is set by the stellar accretion rate $\dot{M}_{\text {acc }}$, for which we took the representative $\dot{M}_{\text {acc }}$ for four stellar masses, $M_{*}=[0.1,0.2,0.32,1.0] \mathrm{M}_{\odot}$, from observations. As the observations show a spread in $\dot{M}_{\text {acc }}$ there should be a similar spread in $M_{\text {disk }}(t=0)$. Changing the initial disk mass would, to first order, move the model curves to the left or right in Figure 7 While the gas masses of both the models and observations can be varied to some degree, changing the gas masses alone cannot explain the order of magnitude difference between the ${ }^{13} \mathrm{CO}$ fluxes of models and the observations.

The assumption of viscous evolution: Throughout this work we have assumed that protoplanetary disks evolve viscously and calculated the time evolution of ${ }^{13} \mathrm{CO}$ and $\mathrm{C}^{18} \mathrm{O}$ 3-2 line fluxes based on this premise. However, it is also possible disk evolution is instead driven by magnetic disk winds. While a quantitative analysis of this scenario is beyond the scope of this work, we can discuss the expected differences. In our models we saw that the ${ }^{13} \mathrm{CO}$ fluxes, and to some degree the $\mathrm{C}^{18} \mathrm{O}$ fluxes, increase with 
time while the disk mass instead decreases with time. This is attributed to a combination of the ${ }^{13} \mathrm{CO}$ emission being mostly optically thick and the disk viscously spreading as it evolves. If disk evolution is instead driven by disk winds the disk is not expected to spread out and the ${ }^{13} \mathrm{CO}$ and $\mathrm{C}^{18} \mathrm{O}$ line fluxes will not increase over time. Instead, the ${ }^{13} \mathrm{CO}$ and $\mathrm{C}^{18} \mathrm{O}$ fluxes are expected to remain constant with time while the emission is optically thick. To drive the observed stellar mass accretion the disk mass has to decrease over time. At some point the ${ }^{13} \mathrm{CO}$ and $\mathrm{C}^{18} \mathrm{O}$ emission will become optically thin and the line fluxes will start to decrease over time, similarly to what is seen for $\mathrm{C}^{18} \mathrm{O}$ at $2 \mathrm{Myr}$ in Figure 5. It might take more time for the emission to become optically thin because, in contrast to a viscously spreading disk, the disk mass is not distributed out over an increasingly larger area. Indeed, if disks start out small as suggested by observations (e.g Maury et al. 2019; Tobin et al. 2020), it is possible that the ${ }^{13} \mathrm{CO}$ emission remains optically thick as most of the mass is concentrated in a small area. To test whether disk evolution is driven by disk winds or by viscous spreading, future observations should focus on detecting and resolving the ${ }^{12} \mathrm{CO},{ }^{13} \mathrm{CO}$ and $\mathrm{C}^{18} \mathrm{O}$ emission of the disks that make up the bulk of the disk population of star-forming regions spread over a wide age range.

\section{Conclusions}

In this work we have used the thermochemical code DALI to run a series of viscously evolving disk models with initial disk masses based on observed stellar mass accretion rates. Using these models we examined how CO isotopolog line fluxes, commonly used as to measure disk gas masses, change over time in a viscously evolving disk. We also compared our models to ${ }^{13} \mathrm{CO}$ $J=3-2$ and $\mathrm{C}^{18} \mathrm{O} J=3-2$ observations of disks in the Lupus star-forming region, to investigate if they are consistent with disks evolving viscously. Here we present our conclusions:

- ${ }^{13} \mathrm{CO}$ and $\mathrm{C}^{18} \mathrm{O}$ 3-2 fluxes of viscously evolving disks increase over time due to the lines being optically thick and their optically thick emitting area increasing in size as the disk expands. Only for disks around stars with $M_{*} \leq 0.2 \mathrm{M}_{\odot}$ that evolve with a moderate amount of turbulence $\left(\alpha_{\text {visc }} \geq\right.$ $10^{-3}$ ) the $\mathrm{C}^{18} \mathrm{O}$ 3-2 emission is optically thin throughout the disk and the integrated $\mathrm{C}^{18} \mathrm{O}$ flux thus trace the disk mass.

- Including the conversion of CO through grain-surface chemistry at $<35 \mathrm{~K}$ does not affect the ${ }^{13} \mathrm{CO}$ flux. Initially the $\mathrm{C}^{18} \mathrm{O}$ is also not affected, but from $\sim 1 \mathrm{Myr}$ onward it starts to decrease up to a factor of $\sim 2-3$ at $10 \mathrm{Myr}$. This also ensures that from $\sim 1$ Myr and onward $\mathrm{C}^{18} \mathrm{O}$ 3-2 emission decreases with time in a viscously evolving disk.

- The observed ${ }^{13} \mathrm{CO}$ 3-2 and $\mathrm{C}^{18} \mathrm{O}$ 3-2 line fluxes of the most massive disks $\left(M_{\text {disk }} \gtrsim 5 \times 10^{-3} \mathrm{M}_{\odot}\right)$ in Lupus are consistent to within a factor of two with our viscously evolving disk models where $\mathrm{CO}$ is converted into other species through grain-surface chemistry, assuming a moderate cosmic-ray ionization rate $\zeta_{\mathrm{cr}} \sim 10^{-17} \mathrm{~s}^{-1}$.

- Increasing the cosmic-ray ionization rate to $\zeta_{\text {cr }} \gtrsim 5 \times 10^{-17}$ $10^{-16} \mathrm{~s}^{-1}$ decreases the $\mathrm{C}^{18} \mathrm{O}$ fluxes to within a factor of $\sim 2$ of the observed upper limits for disks in Lupus with $M_{\text {disk }} \lesssim 5 \times 10^{-3} \mathrm{M}_{\odot}$. Reproducing the stacked $\mathrm{C}^{18} \mathrm{O}$ upper limit observed in Lupus requires a lower average abundance, which could be obtained with efficient vertical mixing.

- Our models overpredict the observed ${ }^{13} \mathrm{CO} 3-2$ fluxes by a factor of $10-30$ for most disks with $M_{\text {disk }} \lesssim 5 \times 10^{-3} \mathrm{M}_{\odot}$ because the ${ }^{13} \mathrm{CO} 3-2$ emission originates from a layer at $z / r \sim 0.25-0.4\left(=2.5-4 \times h_{c}\right.$ for our models $)$, which is much higher up than the region where $\mathrm{CO}$ can be efficiently converted into other species $(z / r \lesssim 0.15)$.

- Reproducing the ${ }^{13} \mathrm{CO} 3-2$ observations in Lupus requires both efficient vertical mixing and a higher cosmic-ray ionization rate, $\zeta_{\mathrm{cr}} \sim 5 \times 10^{-17}-10^{-16} \mathrm{~s}^{-1}$. Alternatively, the observations can be explained if less massive $\left(M_{\text {dust }} \lesssim\right.$ $\left.3 \times 10^{-5} \mathrm{M}_{\odot}\right)$ disks are either much flatter and colder or much smaller $\left(R_{\mathrm{CO}, 90 \%} \sim 40-70 \mathrm{AU}\right)$ than their more massive counterparts.

Our models show that the observed $\mathrm{C}^{18} \mathrm{O}$ fluxes in Lupus are consistent with these disks having evolved viscously, if $\mathrm{CO}$ has been converted into other species under a high cosmic-ray ionization rate. The observed ${ }^{13} \mathrm{CO}$ fluxes are also consistent with this picture, provided that the material in the disk is also well mixed vertically. However, alternative explanations for the low observed ${ }^{13} \mathrm{CO}$ fluxes such as the disks being colder or smaller than assumed cannot be discarded based on current observations. Deeper observations that resolve the $\mathrm{CO}$ isotopolog emission of low mass disks are needed to conclusively demonstrate whether these disks are evolving viscously. Observing the products into which $\mathrm{CO}$ is being converted will be difficult as they are either frozen out or their emission lines lie in the infrared. However, with the James Webb Space Telescope it will be possible to search for $\mathrm{CO}_{2}$ and $\mathrm{CH}_{3} \mathrm{OH}$ ice at intermediate disk layers of edge-on disks or for $\mathrm{CO}_{2}$ gas in the inner disk (see, e.g., Bosman et al. (2017); Anderson et al. (2021) for a more detailed discussion).

Acknowledgements. We thank the referee for the useful comments that helped improve the manuscript. LT and MRH are supported by NWO grant 614.001.352. ADB acknowledges support from NSF Grant\#1907653 and NASA grant XRP 80NSSC20K0259. GR acknowledges support from the Netherlands Organisation for Scientific Research (NWO, program number 016.Veni.192.233). GR also acknowledges an STFC Ernest Rutherford Fellowship (grant number ST/T003855/1). Astrochemistry in Leiden is supported by the Netherlands Research School for Astronomy(NOVA). All figures were generated with the PYTHON-based package MATPLOTLIB (Hunter 2007). This research made use of Astropy ${ }^{2}$ a community-developed core Python package for Astronomy (Astropy Collaboration et al. 2013 2018).

\section{References}

Aikawa, Y., Umebayashi, T., Nakano, T., \& Miyama, S. M. 1997, The Astrophysical Journal Letters, 486, L51

Alcalá, J., Manara, C., Natta, A., et al. 2017, Astronomy \& Astrophysics, 600, A20

Alcalá, J., Natta, A., Manara, C., et al. 2014, Astronomy \& Astrophysics, 561, A2

ALMA Partnership, Brogan, C. L., Pérez, L. M., et al. 2015, ApJ, 808, L3

Anderson, D. E., Blake, G. A., Cleeves, L. I., et al. 2021, arXiv e-prints, arXiv:2101.03182

Andrews, S. M., Terrell, M., Tripathi, A., et al. 2018, ApJ, 865, 157

Andrews, S. M., Wilner, D. J., Espaillat, C., et al. 2011, ApJ, 732, 42

Andrews, S. M., Wilner, D. J., Zhu, Z., et al. 2016, The Astrophysical Journal Letters, 820, L40

Ansdell, M., Williams, J. P., Manara, C. F., et al. 2017, AJ, 153, 240

Ansdell, M., Williams, J. P., Trapman, L., et al. 2018, ApJ, 859, 21

Ansdell, M., Williams, J. P., van der Marel, N., et al. 2016, The Astrophysical Journal, 828, 46

Armitage, P. J. 2015, ArXiv e-prints [arXiv: 1509. 06382]

Astropy Collaboration, Price-Whelan, A. M., Sipőcz, B. M., et al. 2018, AJ, 156, 123

Astropy Collaboration, Robitaille, T. P., Tollerud, E. J., et al. 2013, A\&A, 558, A33

Avenhaus, H., Quanz, S. P., Garufi, A., et al. 2018, ApJ, 863, 44

Bailer-Jones, C. A. L., Rybizki, J., Fouesneau, M., Mantelet, G., \& Andrae, R. 2018, AJ, 156, 58

Balbus, S. A. \& Hawley, J. F. 1991, ApJ, 376, 214

2 http://www.astropy.org 
Balbus, S. A. \& Hawley, J. F. 1998, Reviews of Modern Physics, 70, 1

Barenfeld, S. A., Carpenter, J. M., Ricci, L., \& Isella, A. 2016, The Astrophysical Journal, 827, 142

Barenfeld, S. A., Carpenter, J. M., Sargent, A. I., Isella, A., \& Ricci, L. 2017, The Astrophysical Journal, 851, 85

Benz, W., Ida, S., Alibert, Y., Lin, D., \& Mordasini, C. 2014, in Protostars and Planets VI, ed. H. Beuther, R. S. Klessen, C. P. Dullemond, \& T. Henning, 691

Bergin, E., Cleeves, L. I., Crockett, N., \& Blake, G. 2014, Faraday discussions, 168,61

Bergin, E., Hogerheijde, M., Brinch, C., et al. 2010, Astronomy \& Astrophysics, $521, \mathrm{~L} 33$

Bergin, E. A., Cleeves, L. I., Gorti, U., et al. 2013, Nature, 493, 644

Bergin, E. A., Du, F., Cleeves, L. I., et al. 2016, ApJ, 831, 101

Bergner, J. B., Öberg, K. I., Bergin, E. A., et al. 2019, ApJ, 876, 25

Béthune, W., Lesur, G., \& Ferreira, J. 2017, A\&A, 600, A75

Birnstiel, T., Klahr, H., \& Ercolano, B. 2012, Astronomy \& Astrophysics, 539, A148

Bjerkeli, P., van der Wiel, M. H. D., Harsono, D., Ramsey, J. P., \& Jørgensen, J. K. 2016, Nature, 540, 406

Black, J. H., van Dishoeck, E. F., Willner, S. P., \& Woods, R. C. 1990, ApJ, 358, 459

Booth, R. A., Clarke, C. J., Madhusudhan, N., \& Ilee, J. D. 2017, MNRAS, 469, 3994

Borucki, W. J., Koch, D. G., Basri, G., et al. 2011, ApJ, 736, 19

Bosman, A. D., Bruderer, S., \& van Dishoeck, E. F. 2017, A\&A, 601, A36

Bosman, A. D., Walsh, C., \& van Dishoeck, E. F. 2018, A\&A, 618, A182

Brown, A., Vallenari, A., Prusti, T., et al. 2018, arXiv preprint arXiv:1804.09365

Bruderer, S. 2013, A\&A, 559, A46

Bruderer, S., van Dishoeck, E. F., Doty, S. D., \& Herczeg, G. J. 2012, A\&A, 541, A91

Calahan, J., Bergin, E., Zhang, K., et al. 2020, arXiv e-prints, arXiv:2012.05927

Cardelli, J. A., Meyer, D. M., Jura, M., \& Savage, B. D. 1996, ApJ, 467, 334

Cazzoletti, P., Manara, C. F., Baobab Liu, H., et al. 2019, A\&A, 626, A11

Chiang, E. I. \& Goldreich, P. 1997, ApJ, 490, 368

Cieza, L. A., González-Ruilova, C., Hales, A. S., et al. 2020, arXiv e-prints, arXiv:2012.00189

Cieza, L. A., Ruíz-Rodríguez, D., Hales, A., et al. 2019, MNRAS, 482, 698

Cleeves, L. I., Adams, F. C., \& Bergin, E. A. 2013, The Astrophysical Journal, 772,5

Cleeves, L. I., Bergin, E. A., Qi, C., Adams, F. C., \& Öberg, K. I. 2015, ApJ, 799, 204

Cleeves, L. I., Öberg, K. I., Wilner, D. J., et al. 2018, ApJ, 865, 155

Cox, E. G., Harris, R. J., Looney, L. W., et al. 2017, ApJ, 851, 83

Cridland, A. J., Bosman, A. D., \& van Dishoeck, E. F. 2020, A\&A, 635, A68

de Valon, A., Dougados, C., Cabrit, S., et al. 2020, A\&A, 634, L12

Dodson-Robinson, S. E., Evans, Neal J., I., Ramos, A., Yu, M., \& Willacy, K. 2018, ApJ, 868, L37

Du, F., Bergin, E. A., \& Hogerheijde, M. R. 2015, ApJ, 807, L32

Dubrulle, B., Morfill, G., \& Sterzik, M. 1995, Icarus, 114, 237

Dullemond, C. P., Birnstiel, T., Huang, J., et al. 2018, ApJ, 869, L46

Dullemond, C. P. \& Dominik, C. 2004, A\&A, 421, 1075

Dullemond, C. P. \& Dominik, C. 2005, A\&A, 434, 971

Facchini, S., Birnstiel, T., Bruderer, S., \& van Dishoeck, E. F. 2017, A\&A, 605, A16

Favre, C., Cleeves, L. I., Bergin, E. A., Qi, C., \& Blake, G. A. 2013, ApJ, 776, L38

Fedele, D., van Dishoeck, E. F., Kama, M., Bruderer, S., \& Hogerheijde, M. R. 2016, A\&A, 591, A95

Ferreira, J., Dougados, C., \& Cabrit, S. 2006, A\&A, 453, 785

Furuya, K. \& Aikawa, Y. 2014, ApJ, 790, 97

Garufi, A., Avenhaus, H., Pérez, S., et al. 2020, A\&A, 633, A82

Hartmann, L., Calvet, N., Gullbring, E., \& D’Alessio, P. 1998, ApJ, 495, 385

Heays, A. N., Bosman, A. D., \& van Dishoeck, E. F. 2017, A\&A, 602, A105

Hunter, J. D. 2007, Computing in science \& engineering, 9, 90

Jonkheid, B., Dullemond, C. P., Hogerheijde, M. R., \& van Dishoeck, E. F. 2007 A\&A, 463, 203

Kama, M., Bruderer, S., van Dishoeck, E. F., et al. 2016, A\&A, 592, A83

Kama, M., Folsom, C. P., \& Pinilla, P. 2015, A\&A, 582, L10

Kama, M., Trapman, L., Fedele, D., et al. 2020, A\&A, 634, A88

Kastner, J. H., Qi, C., Gorti, U., et al. 2015, ApJ, 806, 75

Krijt, S., Bosman, A. D., Zhang, K., et al. 2020, arXiv e-prints, arXiv:2007.09517

Krijt, S., Schwarz, K. R., Bergin, E. A., \& Ciesla, F. J. 2018, ApJ, 864, 78

Long, F., Herczeg, G. J., Harsono, D., et al. 2019, ApJ, 882, 49

Long, F., Herczeg, G. J., Pascucci, I., et al. 2017, The Astrophysical Journal, 844 99

Long, F., Pinilla, P., Herczeg, G. J., et al. 2018, ApJ, 869, 17

Loomis, R. A., Cleeves, L. I., Öberg, K. I., Guzman, V. V., \& Andrews, S. M. 2015, ApJ, 809, L25

Lynden-Bell, D. \& Pringle, J. E. 1974, MNRAS, 168, 603
Manara, C. F., Natta, A., Rosotti, G. P., et al. 2020, arXiv e-prints, arXiv:2004.14232

Manara, C. F., Rosotti, G., Testi, L., et al. 2016, A\&A, 591, L3

Manara, C. F., Testi, L., Herczeg, G. J., et al. 2017, A\&A, 604, A127

Maret, S., Maury, A. J., Belloche, A., et al. 2020, arXiv e-prints, arXiv:2001.06355

Maury, A. J., André, P., Testi, L., et al. 2019, A\&A, 621, A76

McClure, M. K., Bergin, E. A., Cleevs, L. I., et al. 2016, AJ

McClure, M. K., Dominik, C., \& Kama, M. 2020, A\&A, 642, L15

Miotello, A., Bruderer, S., \& van Dishoeck, E. F. 2014, A\&A, 572, A96

Miotello, A., Facchini, S., van Dishoeck, E. F., et al. 2019, A\&A, 631, A69

Miotello, A., van Dishoeck, E., Williams, J., et al. 2017, Astronomy \& Astrophysics, 599, A113

Mordasini, C. 2018, Planetary Population Synthesis, 143

Morton, T. D., Bryson, S. T., Coughlin, J. L., et al. 2016, ApJ, 822, 86

Mulders, G. D. \& Dominik, C. 2012, A\&A, 539, A9

Murillo, N. M., Lai, S.-P., Bruderer, S., Harsono, D., \& van Dishoeck, E. F. 2013, A\&A, 560, A103

Öberg, K. I., Guzmán, V. V., Merchantz, C. J., et al. 2017, ApJ, 839, 43

Padovani, M., Ivlev, A. V., Galli, D., \& Caselli, P. 2018, A\&A, 614, A111

Pascucci, I., Testi, L., Herczeg, G., et al. 2016, The Astrophysical Journal, 831, 125

Pinilla, P., Birnstiel, T., Ricci, L., et al. 2012, A\&A, 538, A114

Pontoppidan, K. M., Blake, G. A., \& Smette, A. 2011, ApJ, 733, 84

Pringle, J. E. 1981, ARA\&A, 19, 137

Rab, C., Güdel, M., Padovani, M., et al. 2017, A\&A, 603, A96

Riols, A. \& Lesur, G. 2018, A\&A, 617, A117

Rosotti, G. P., Clarke, C. J., Manara, C. F., \& Facchini, S. 2017, MNRAS, 468, 1631

Schwarz, K. R., Bergin, E. A., Cleeves, L. I., et al. 2016, AJ, 823, 91

Schwarz, K. R., Bergin, E. A., Cleeves, L. I., et al. 2018, ApJ, 856, 85

Schwarz, K. R., Bergin, E. A., Cleeves, L. I., et al. 2019, ApJ, 877, 131

Semenov, D. \& Wiebe, D. 2011, ApJS, 196, 25

Shakura, N. I. \& Sunyaev, R. A. 1973, A\&A, 24, 337

Tabone, B., Cabrit, S., Bianchi, E., et al. 2017, A\&A, 607, L6

Terwisscha van Scheltinga, J., Hogerheijde, M. R., Cleeves, L. I., et al. 2020, arXiv e-prints, arXiv:2011.07073

Tobin, J. J., Sheehan, P. D., Megeath, S. T., et al. 2020, ApJ, 890, 130

Trapman, L., Facchini, S., Hogerheijde, M. R., van Dishoeck, E. F., \& Bruderer, S. 2019, A\&A, 629, A79

Trapman, L., Miotello, A., Kama, M., van Dishoeck, E. F., \& Bruderer, S. 2017, A\&A, 605, A69

Trapman, L., Rosotti, G., Bosman, A. D., Hogerheijde, M. R., \& van Dishoeck, E. F. 2020, arXiv e-prints, arXiv:2005.11330

Turner, N. J., Fromang, S., Gammie, C., et al. 2014, Protostars and Planets VI, 411

Tychoniec, Ł., Manara, C. F., Rosotti, G. P., et al. 2020, A\&A, 640, A19

Tychoniec, Ł., Tobin, J. J., Karska, A., et al. 2018, ApJS, 238, 19

van Boekel, R., Henning, T., Menu, J., et al. 2017, ApJ, 837, 132

van der Marel, N., Williams, J. P., Ansdell, M., et al. 2018, The Astrophysical Journal, 854, 177

van Dishoeck, E. F. \& Black, J. H. 1988, ApJ, 334, 771

van 't Hoff, M. L. R., van Dishoeck, E. F., Jørgensen, J. K., \& Calcutt, H. 2020, A\&A, 633, A7

van Terwisga, S. E., van Dishoeck, E. F., Ansdell, M., et al. 2018, A\&A, 616, A88

van't Hoff, M. L. R., Harsono, D., Tobin, J. J., et al. 2020, ApJ, 901, 166

Vernet, J., Dekker, H., D’Odorico, S., et al. 2011, A\&A, 536, A105

Visser, R., van Dishoeck, E. F., \& Black, J. H. 2009, A\&A, 503, 323

Weingartner, J. C. \& Draine, B. 2001, The Astrophysical Journal, 548, 296

Willacy, K., Langer, W., Allen, M., \& Bryden, G. 2006, ApJ, 644, 1202

Williams, J. P., Cieza, L., Hales, A., et al. 2019, The Astrophysical Journal, 875, L9

Wilson, T. L. \& Rood, R. 1994, ARA\&A, 32, 191

Winn, J. N. \& Fabrycky, D. C. 2015, ARA\&A, 53, 409

Woitke, P., Kamp, I., \& Thi, W.-F. 2009, A\&A, 501, 383

Woodall, J., Agúndez, M., Markwick-Kemper, A., \& Millar, T. 2007, Astronomy \& Astrophysics, 466, 1197

Yen, H.-W., Koch, P. M., Manara, C. F., Miotello, A., \& Testi, L. 2018, A\&A, 616, A100

Yu, M., Evans, Neal J., I., Dodson-Robinson, S. E., Willacy, K., \& Turner, N. J. 2017, ApJ, 841, 39

Yu, M., Willacy, K., Dodson-Robinson, S. E., Turner, N. J., \& Evans, Neal J., I. 2016, ApJ, 822, 53

Zhang, K., Bergin, E. A., Schwarz, K., Krijt, S., \& Ciesla, F. 2019, ApJ, 883, 98 Zhu, Z. \& Stone, J. M. 2018, ApJ, 857, 34 
Appendix A: Model ${ }^{13} \mathrm{CO}$ and $\mathrm{C}^{18} \mathrm{O} J=3-2$ intensity profiles

Appendix B: Model ${ }^{13} \mathrm{CO}$ and $\mathrm{C}^{18} \mathrm{O} J=3-2$ integrated fluxes

Appendix C: Model ${ }^{13} \mathrm{CO}$ and $\mathrm{C}^{18} \mathrm{O} J=2-1$ fluxes

Appendix D: Comparing maximum CO conversion models to observed $\mathrm{CO}$ isotopolog line fluxes 


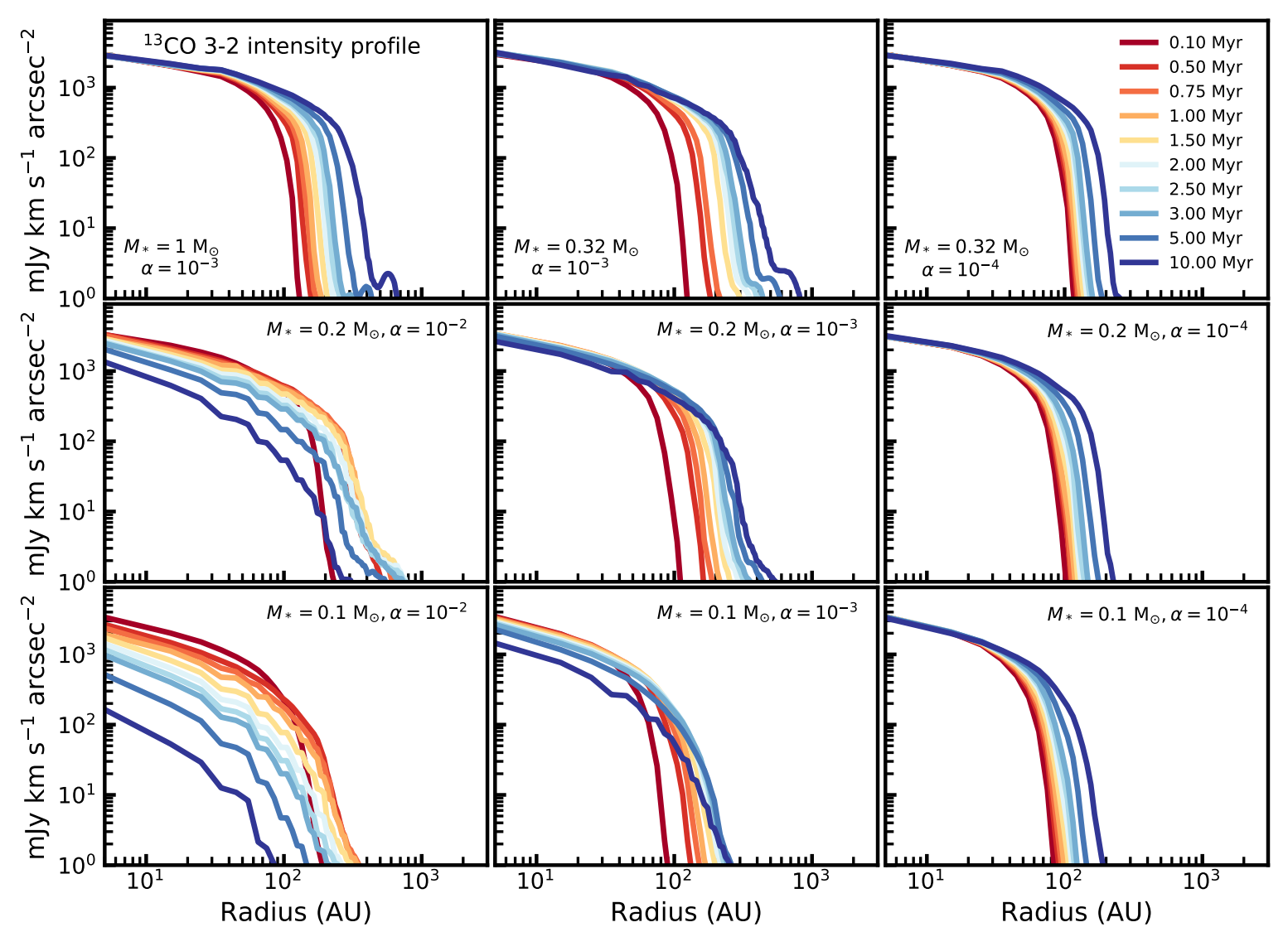

Fig. A.1: Time evolution of the ${ }^{13} \mathrm{CO} J=3-2$ intensity profiles of our models, where colors denote different time steps. Each panel shows a different $M_{*}$ and $\alpha_{\text {visc }}$. 


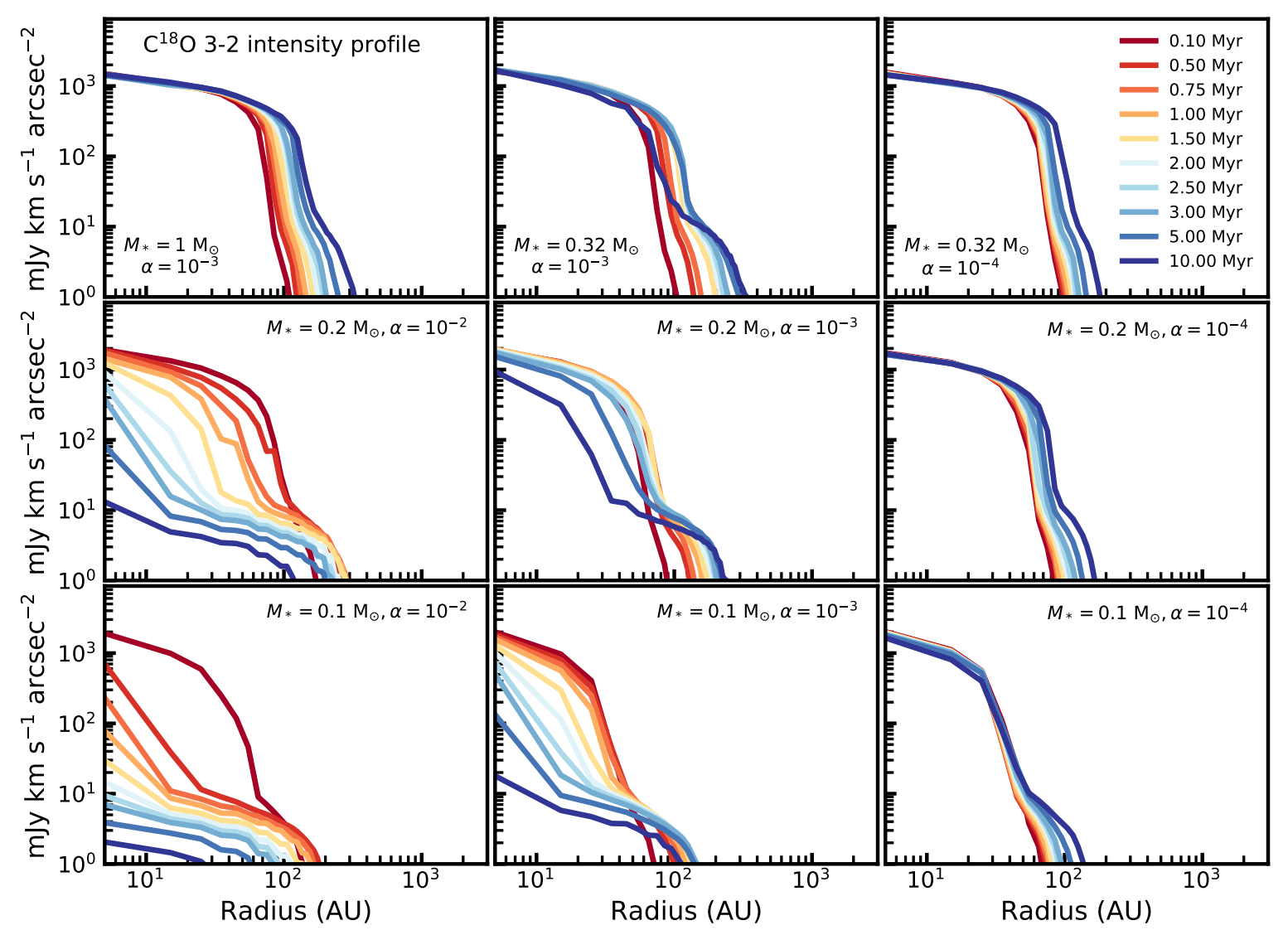

Fig. A.2: Time evolution of the ${ }^{13} \mathrm{CO} J=3-2$ intensity profiles of our models, where colors denote different time steps. Each panel shows a different $M_{*}$ and $\alpha_{\text {visc. }}$. 

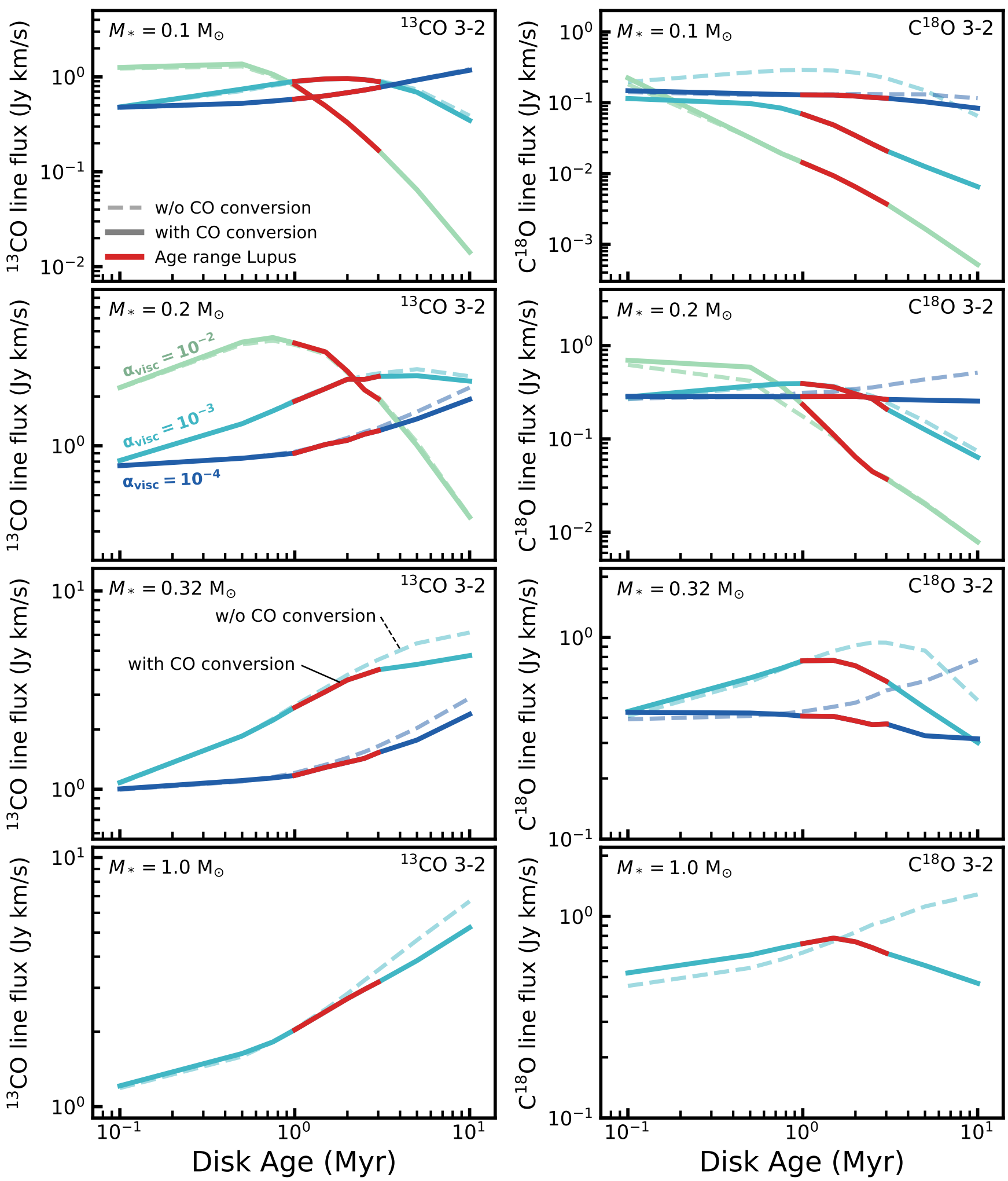

Fig. B.1: As Figure 6, but showing the ${ }^{13} \mathrm{CO}$ (left) and $\mathrm{C}^{18} \mathrm{O}$ (right) $J=3-2$ fluxes for all models. Colors indicate different $\alpha_{\text {visc }}$. Models with and without $\mathrm{CO}$ conversion through grain-surface chemistry are shown with solid and dashed lines, respectively. The age range for disks in Lupus is highlighted in red. Few models show a significant decrease in ${ }^{13} \mathrm{CO}$ line fluxes, except for models with $\alpha_{\text {visc }}=10^{-2}$ that have low disk masses. The $\mathrm{C}^{18} \mathrm{O}$ line fluxes do show a decrease with age, especially at later disk ages. 

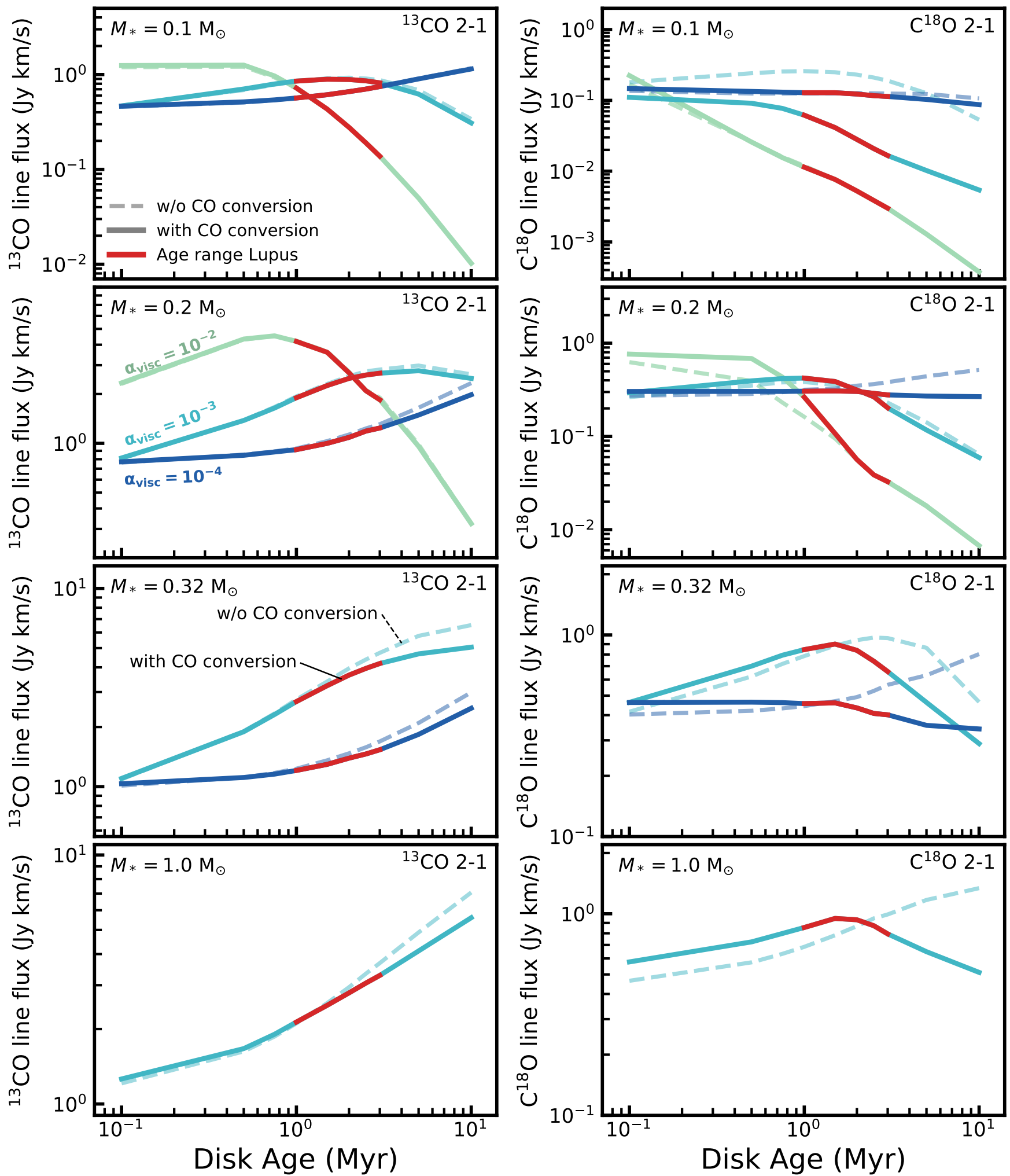

Fig. C.1: As Figure B.1, but showing the ${ }^{13} \mathrm{CO}$ (left) and $\mathrm{C}^{18} \mathrm{O}$ (right) $J=2-1$ fluxes for all models. We note that the range of the $y$-axes are the same as for Figure B.1 

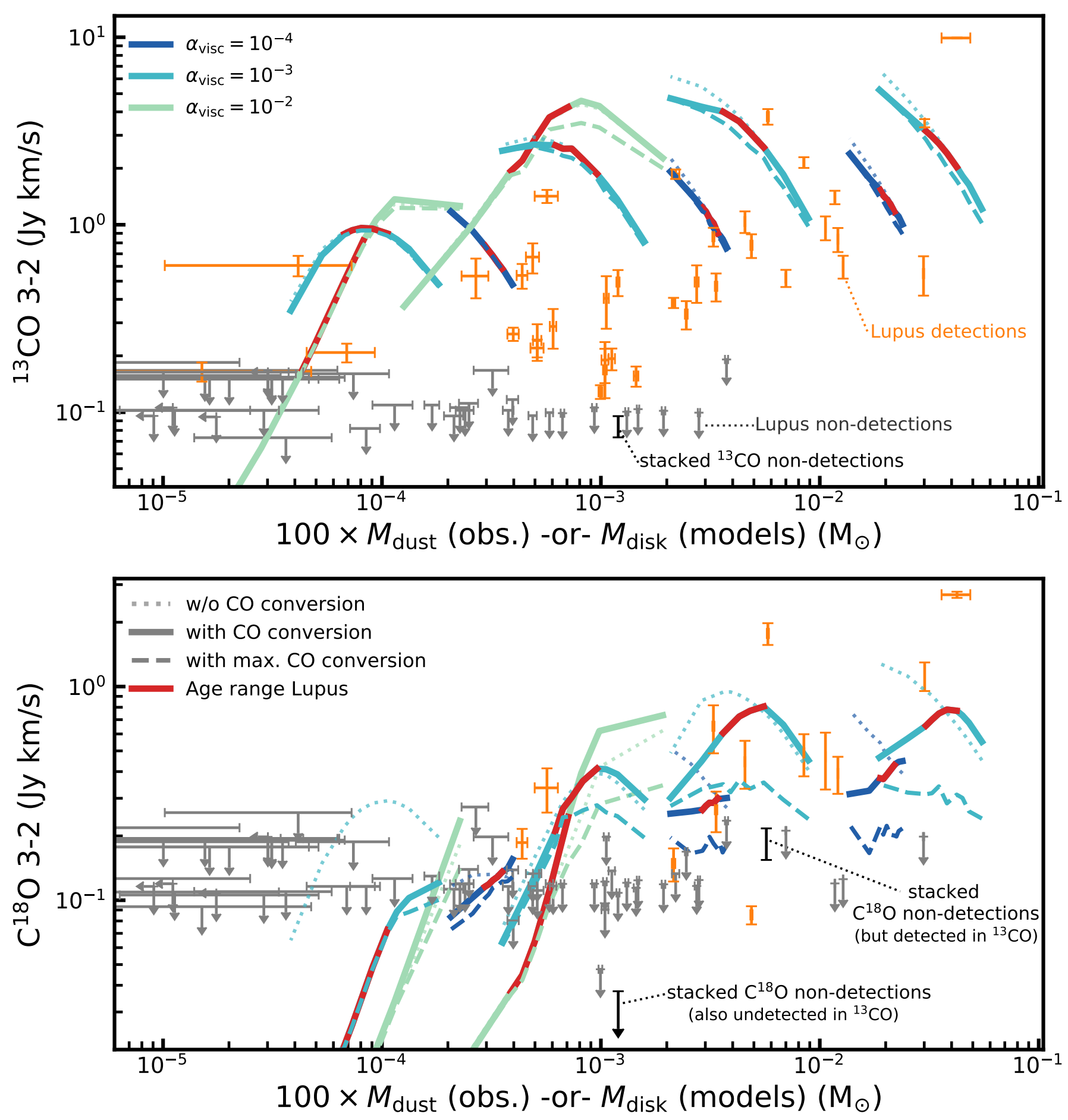

Fig. D.1: As Figure 6, but now showing three sets of models. The dotted lines show the ${ }^{13} \mathrm{CO}$ (top) and $\mathrm{C}^{18} \mathrm{O}$ (bottom) $J=3-2$ line fluxes for models with standard DALI chemistry, that is, without CO conversion. The solid lines show line fluxes for the models where CO has been chemically converted into other species, as described in Section 2.3.2. The dashed lines show line fluxes for models with maximal $\mathrm{CO}$ conversion, where we have removed all $\mathrm{CO}$ in the region where $\mathrm{CO}$ conversion through grain-surface chemistry occurs (see also Section 8 ). Observations in Lupus are shown in orange if detected and gray if an upper limit (Ansdell et al. 2016; Yen et al. 2018). Stacked non-detections are shown in black (see Section 3.3. 